\title{
Fluid-structure interaction for vascular flows: from supercomputers to laptops
}

\author{
Claudia M. Colciago, Simone Deparis and Davide Forti
}

\begin{abstract}
There exists several models for the simulation of vascular flows; they span from simple circuit models, to full three dimensional ones which take into account detailed features of the blood and of the arterial wall. Each model comes with benefits and drawbacks, the main denominator being a compromise between detailed resolution requirements versus computational time.
\end{abstract}

We first present a fluid-structure interaction computational model where both the fluid and the structure are three dimensional; in particular, the fluid includes modeling of large eddies by the variational multiscale method. After time and space discretisations carried out by finite differences and finite elements, respectively, we set up a parallel solver based on domain decomposition and a FaCSI preconditioner. These simulations allows to capture details of the flow dynamics and of the structure deformation even in the transitional regime characterizing the hemodynamics in the aorta. To complete a simulation of one heartbeat with 35 millions of degrees of freedom on 2048 cores it takes roughly 10 hours.

We then reduce both the model and its numerical complexity. The structural model is simplified to a two dimensional membrane located at the fluid-structure interface and the fluid computational domain is fixed. For a fixed geometry and mesh, these assumptions allow to apply proper orthogonal decomposition and generate a space discretisation which has only few dozen degrees of freedom. It is then possible to perform the simulation of one hearbeat on a laptop in less than one second.

The modeling and numerical reductions allows therefore a dramatic reduction of the computational time. However, the price to pay comes, on the one hand, in terms of the preparation of a reduced basis specific to the patient and the geometry of the vessel and, on the other hand, with a detriment of certain quantities of interest. For example, when using a finite element discretization with 9 millions of degrees of freedom, the offline part takes about 12 hours on 720 cores for the example provided in this work; in this case, the flow profiles in the aorta are pretty close to the full three dimensional model, but the wall shear stress is overestimated (although it follows the same temporal patterns).

Keywords. Fluid structure interaction, reduced order modeling, hemodynamics, high performance computing, algorithms for FSI, preconditioners for FSI.

AMS classification. 65M60, 65M55, 76Z05, 76D05, 35Q30, 35Q35, 92C10.

We gratefully acknowledge the CSCS for providing us the CPU resources under project ID s635. ERC Advanced grant N.227058 MATHCARD, SNF (no. 140184) and PASC, 


\section{Introduction}

The complexity of the cardiovascular system poses numerous challenges for its biological and mathematical modeling, numerical discretization and simulation. The study of the cardiovascular system is mainly motivated by the large amount of pathologies and deaths related to cardiovascular diseases. Heart attacks and strokes are responsible of half of human deaths in the developed countries. Two of the main cardiovascular diseases are atherosclerosis and aneurysms. Atherosclerosis is an accumulation of fatty material, fibrous elements and calcium located in the intima which is the structural layer of the arterial wall that is in direct contact with the blood flow. Several studies have shown that the mechanical stimuli of the blood flow on the arterial wall are strictly related to the developing of atherosclerosis [81, 108, 69, 80]. Another example of a widespread vascular disease is represented by aneurysms. An aneurysm is a gradual stretching of the arterial wall that becomes thinner and weaker. Although aneurysms can develop in all the arteries of the cardiovascular system, they are more likely to appear in cerebral arteries and in the ascending and abdominal parts of the aorta. It has been shown that pathogenesis of aortic aneurysm can be triggered by mechanical stimuli, such as low or oscillatory wall shear stress, or also by regions featuring flow recirculations or stagnations $[19,97,82]$. It is evident that the study of hemodynamics is of primary importance from the clinical standpoint, hence the interest in modeling these problems from the mathematical point of view and in numerical simulations.

The blood flow and the arterial wall are modeled by continuum mechanics; their interaction is expressed by the action-reaction principle (equilibrium of stresses) and by the fluid sticking to the wall (continuity of displacements and velocities). The modeling, the discretization and the design of numerical schemes to solve efficiently this coupled Fluid-Structure Interaction (FSI) problem are still challenging tasks and major topics of ongoing research. Despite the fact that cardiovascular FSI problem have been studied for almost two decades, their intrinsic complexity keeps the debate open about the set up of appropriate models and algorithms. Numerical simulations of such complex system may provide detailed and quantitative information to improve the understanding of the underlying complex physical phenomena, thus helping and supporting the development of more effective clinical treatments without using invasive techniques.

The mathematical models of the constituents of the cardiovascular system such as the arterial wall and blood should be able to reproduce their most important physiological features and the biological interactions among them. The vascular system is composed by a network of vessels in which blood flows under the stimuli of a quasi periodic pump, the heart. Blood is a heterogeneous medium, composed by a particulate phase and a liquid one. The fluid part is called plasma and it behaves like water. Despite its heterogeneity, as a common assumption, blood in large vessels is considered as a continuous media since the dimension of the particles is of orders of magnitude smaller than the diameter of the vessel. Concerning the viscous properties of blood, 
at low shear rates it shows a non-Newtonian behavior: in small vessel like capillaries, blood particles tend to form aggregates which affect the fluid viscosity. Nevertheless, in large arteries with diameter greater than $0.3 \mathrm{~cm}$, it is widely accepted to assume a Newtonian behavior. Since our goal is to focus on large vessels such as the aorta (about $3 \mathrm{~cm}$ diameter) and its main bifurcations, we also choose a constant value for blood viscosity [92].

The resulting mathematical model is expressed by the Navier-Stokes equations [92, 44]. The other component of our physical system is the arterial wall. Vessels are compliant pipes that convey the blood to all the organs of the body, they are complex living tissues that react and interact with the blood flow depending on several factors. Due to the difficulty of performing in vivo experiments on vessel tissues, the rheological behaviour of the arterial wall is still an ongoing topic of research [112]. Several models have been proposed featuring increasing level of complexity. In the reference literature both linear and nonlinear models have been used to perform FSI simulations of the cardiovascular system, featuring both isotropic and anisotropic properties (see e.g. $[118,96,119,62])$. Some models are enhanced by multi-layered structure, by the presence of fibers or of an intraluminal thrombus (see e.g. [48, 36, 116]). Also damage and growth models raised the interests of researchers, in particular for their relevance in pathological conditions (see e.g. $[63,66,71]$ ). Not only the choice of the constitutive model is challenging, but also the assessment and the tuning of the mechanical parameters that show a significant variation among both intra- and inter- patient conditions. As mathematical model for the artery we choose a quasi-incompressible continuous elastic material governed by an iperbolic partial differential equation.

In this paper we focus on the comparisons between different numerical choices rather than comparing them with experimental results. Thus, for the sake of our presentation, we will consider a rather simplistic structural model featuring a linear elastic and isotropic constitutive law, being aware that our numerical output can be distant from the in vivo biomechanical behavior.

The coupled fluid-structure interaction problem can not be solved analytically and so it has to be studied by means of experiments or by numerical simulations; we are interested in the latter.

On the modeling side, a distinction between the different methodologies to deal with fluid-structure interaction problems comes from the choice of the frame of reference in which the problem itself is formulated. We choose to describe the structure in a Lagrangian reference and the fluid equations using an Arbitrary Lagrangian-Eulerian (ALE) frame of reference $[109,93,122,64,14,45,49,73,26,15]$ which requires a new equation for the fluid domain motion. Another possibility would consist in using a space-time formulation of the FSI problem. It involves a discretization of the computational domain in time slabs, and each solution in a time slab is then computed sequentially (see $[113,114,111,15]$ for a complete overview on this formulation). It is also possible to formulate the FSI problem in a fully Eulerian frame of reference $[25,124,103]$, i.e. on a fixed fluid domain; in this case, one needs to keep track 
of the position of the fluid-structure interface. In [38, 101, 102], a fully Eulerian formulation of FSI is used in two dimensions to avoid the degeneration of the ALE mapping and to facilitate mesh adaptivity. Yet, another approach is the immersed boundary method, where the fluid is written in Eulerian coordinates, while the structure is still in a Lagrangian frame of reference $[98,89,18]$. A further alternative approach to ALE methods is based on the use of the Extended Finite Element method (XFEM) $[53,123,87]$.

The use of an ALE formulation for the fluid, together with a Lagrangian frame for the structure, yields an FSI problem that is composed by three subproblems, namely the fluid problem, which allows for the computation of the velocity and pressure inside the fluid domain, the solid problem, which describes the deformation of the vessel wall, and the so-called geometry problem, which accounts for the moving computational fluid domain. A peculiarity in biomechanics is the similar density of the fluid and the structure, which, combined with incompressible flows, leads to increased numerical effort due to an added-mass effect [22, 6, 120, 121]. Because of the added mass effect, staggered methods, where the fluid and the solid are explicitly coupled, are numerically unstable. Extensive work has been carried out on the development of algorithms for the solution of time-dependent FSI problems in the framework of biomechanics. The approaches are typically categorized either as segregated or as monolithic schemes although the distinction is not always straightforward. Segregated schemes can range from simple, loosely coupled fixed point iterations to schemes which still use segregated solvers but apply a much stronger coupling. Monolithic schemes may include block preconditioners constructed from segregated solvers as well as preconditioners for the fully coupled problem which are not constructed from separate solvers. In monolithic schemes all the unknowns of the FSI problem are solved simultaneously. Monolithic algorithms were investigated, e.g., in [88, 64, 14, 73, 49, 12, 127, 26]. In [33], a comparison of the performance of strongly coupled Steklov-Poincaré algorithm using the Dirichlet-Neumann, Neumann-Dirichlet, and Neumann- Neumann preconditioners with a monolithic approach preconditioned by a Dirichlet-Neumann preconditioner is reported.

A modular approach to solve the FSI problem would consist in dealing with the three problems separately, but treat their coupling implicitly. For example, one can consider the FSI problem using different type of interface conditions (Dirichlet-Neumann [79, 86, 75, 76, 77], Robin-Robin [6, 5, 95], Robin-Neumann [42], Neumann-Neumann [33], FETI [30], etc. [117]) to ensure the coupling. A comparison of different coupling schemes for FSI problems in hemodynamics is carried out in [73]. A further approach makes use of a Steklov-Poincaré formulation [29] to enforce the coupling on the fluidstructure interface.

The way the coupled set of equations describing the FSI phenomena are discretized in time induces further distinctions. One approach may consist in dealing with all the nonlinearities of the problem in an implicit mode, leading to the so-called Fully Implicit (FI) strategy $[41,59,115,35,14,60,12,73,49,127]$. Although this approach is 
the most stable, it is also the most expensive choice. A large variety of alternative time discretizations can be devised. For instance, a Convective Explicit time discretization is used in [26], in which the nonlinear convective term in the unsteady Navier-Stokes equations is treated using a semi-implicit approach. Further, a Geometry-Convective Explicit discretization is proposed in $[6,4,26]$ wherein the moving geometry is taken at the previous time step and the convective term is treated semi-implicitly. A possible approach to deal with the nonlinearity makes use of the Aitken accelerated fixed point algorithm, see e.g. [79, 22, 74, 75, 6, 29, 86]. In this way each fixed point iteration requires one residual evaluation. Otherwise the time discretized problem can be linearized via the Newton method, either considering the full Jacobian matrix, as in $[49,41,115]$, or neglecting some of its contributions, as in [50, 28, 59]. Each nonlinear Newton iteration requires a solve of the linearized subproblems. Thus the cost of each nonlinear iteration corresponds to the cost of one residual evaluation plus a variable number of solutions of the linearized subproblems.

After spatial and temporal discretizations, one of the key aspects which characterizes the different methodologies is the choice of the preconditioner. For instance, relying on monolithic schemes, one may use block preconditioners built upon physicspecific solvers as well as preconditioners for the fully coupled problem which are not constructed from separate solvers. Several strategies have been proposed in literature, see e.g., [59, 6, 8, 49, 12, 26, 127]. Overlapping Schwarz methods within monolithic approaches were studied [26] in different regimes of severity of the added-mass effect confirming successful results for 2D already reported in [12]. A monolithic FSI approach coupling nonlinear hyperelastic solid models with Navier-Stokes equations is presented in [64], considering the incompressible case for the solid. A block preconditioner with Schur complements for the monolithic system is presented in [68]. A scalable monolithic solver for an FSI problem coupling blood flow with a conforming arterial wall in two dimensions is presented in $[11,12]$. There, a Newton scheme with an explicitly computed Jacobian is applied; see also [41, 14, 12]. For the solution of the FSI linearized systems, in [12] the authors use a hybrid multilevel Schwarz preconditioner which uses restricted additive Schwarz on the fine level and multiplicative Schwarz on the coarse level. The parallel Newton-Krylov-Schwarz approach for the monolithic system is extended to three dimensions in [127], and scalability is shown for up to three thousand processors. Parallel algebraic multigrid preconditioners have recently been applied to fully monolithic ALE formulations of FSI problems in the setting of biomechanics, see, e.g., [49, 125]. Specifically, in [49], the authors propose two preconditioners that apply algebraic multigrid techniques to the linearized monolithic FSI system obtained after spatial and time discretizations.

It is clear that the numerical solution of full three dimensional coupled FSI problems is extremely challenging and, as we discussed above, requires ad hoc strategies to lower computational costs. Despite the fact that advanced parallel solvers and preconditioners successfully managed to decrease the costs of numerical simulations, we are still far from having a real time resolution of FSI problems using a finite element 
method. The intrinsic complexity of the system and the fine unstructured meshes yield computational costs that are orders of magnitude bigger than the simulated physical time: for the solution of few heartbeats (order of seconds), several hours of CPU time on supercomputers are required. Also in terms of memory the FSI problem is expensive: the dimensions of the algebraic system and the discretized solutions are of orders of millions of degrees of freedom and they need a suitable amount of memory to be stored and manipulated. It is therefore necessary to use supercomputers and be able to solve the discretized model in parallel.

A major issue arises when we consider the final goal of these numerical simulations.

The desired features of numerical tools which may be used in everyday clinical practice includes reliability, parameter tuning and real time computations. Indeed, numerical tools should allow specialists to compute accurate approximations of target medical outputs together with the possibility of tuning patient-specific physical parameters. Moreover, these computations should be performed in a small amount of time and, ideally, using a limited amout of computational resources (e.g. using a personal computer). These challenges has driven a reasearch stream of numerical and applied mathematics in the latest years: a great effort has been devoted to the study of numerical reduction techniques. These methods aims at dramatically lowering the computational costs through a likewise dramatical decrement of the dimension of the approximation spaces: numerical reduction techniques aims at describing the approximated solution using hundreds of degrees of freedom versus the millions required when using a high fidelity approximation like the finite element one.

Our choice to perform numerical reduction relies on Proper Orthogonal Decomposition (POD) for parametrized PDEs, i.e., partial differential equations or system of equations that depend on pre-selected physical and/or geometrical parameters. In cardiovascular applications, for example, physical parameters may be the mechanical properties of the structure like the young modulus, or the coefficients that affect the boundary conditions [58] like the average inlet flow rate or outlet resistance and compliance coefficients [17]. In scientific research many efforts have also been devoted to the investigation of geometrical parameters, like the inflow angle or the morphology of the domain itself, e.g. in [78, 105, 34, 67, 85, 52].

The POD allows to select a set of basis functions which allows to well approximate the manifold generated by the parameter dependent solutions. In practice, suitable operators are built to project the high fidelity functions onto the low dimensional discretization space. The resulting reduced order model can thus be solved with low computational costs in a many query context, i.e. for different values of the model parameters. To better quantify the efficiency of a reduced order model, it is in general necessary to split the computational costs into two stages: an offline stage, expensive but performed once for the construction of the projection operators, and an online stage that is very rapid, in which reduced approximated solutions are computed for several realizations of the parameters.

For a detailed presentation of reduced order model we adress the interested reader 
to the recent books $[61,99]$. A lot of interest has been devoted to discretization reduction techniques for parametrized PDEs problems in realistic applications (e.g. [100]). Recently, increasing attention has been devoted in particular to haemodynamics and cardiovascolar applications (see e.g. [9, 10, 21, 17, 57, 84, 106, 56, 20]).

\subsection{Outline}

The paper is organized as follows. In Section 2 we introduce the fluid and structure three dimensional models, their coupling, as well as the finite element discretization and the resolution algorithm; the resulting approximation is highly accurate and the computational effort very expensive and requiring parallel solvers. Section 3 presents a possible reduced model for the structure mechanics and the integration of the structure variable into the fluid ones. This allows for a numerical reduction using POD as shown in Section 4; the resulting system is very small and the computational effort can be split into two separate steps: an offline one, requiring the resolution of finite element problems, a little less expensive than the full three-dimension model, and an online one, very cheap. The proposed methods are tested on a geometry including the whole aorta and few additional ramifications, see Section 5; we show numerical results, including comparisons between the full three dimensional model and the reduced one.

\section{Modeling fluid-structure interaction in moving domains}

We are interested in simulating the hemodynamics in a piece of artery. Our model needs to account for the moving walls and at the same time focus on the region of interest. A natural choice for the structure frame of reference is the Lagrangian one. It allows for describing exactly the piece of artery we are interested in. The choice of the fluid frame of reference needs a little more care. If on one side it has to follow the wall movement, hence a Lagrangian frame of reference would fit well, on the other the the fluid leaves the piece of artery after a short time, therefore an Eulerian frame would be advisable. The Arbitrary Lagrangian Eulerian frame of reference allows to account for both the constraints: near the arterial wall, the fluid computational domain sticks to the wall, whilst at the inlet and outlet sides the domain stays fixed.

\subsection{Fluid Structure Interaction modeling}

Let $\widehat{\Omega}_{f}$ and $\widehat{\Omega}_{s}$ be the fluid and solid domains, respectively, in their reference configuration. We denote by $\widehat{\Gamma}=\partial \widehat{\Omega}_{f} \cap \partial \widehat{\Omega}_{s}$ the fluid-structure interface. The initial time is set equal to zero and the final time is denoted by $T$. At any time $t \in[0, T]$, we presume that the current fluid domain configuration $\Omega^{f}(t)$ can be retrieved from $\widehat{\Omega}_{f}$ 
by the Arbitrary Lagrangian Eulerian (ALE) mapping

$$
\begin{aligned}
\mathcal{A}_{t}: \widehat{\Omega}_{f} & \rightarrow \Omega_{f}(t) \\
\widehat{\mathbf{x}} & \mapsto \mathcal{A}_{t}(\widehat{\mathbf{x}})=\widehat{\mathbf{x}}+\widehat{\mathbf{d}}_{f}(\widehat{\mathbf{x}}),
\end{aligned}
$$

where $\widehat{\mathbf{d}}_{f}$ represents the displacement of the fluid domain. The ALE mapping does not represent any physical quantity as it is a mathematical construct. We will need to construct a discrete version which is needed to describe the displacement and velocity of each mesh point. In fact, the use of the ALE formulation allows an arbitrary reconstruction of the volumetric computational grid of $\Omega_{f}(t)$ from the one of the domain boundary $\partial \Omega_{f}(t)$. For the sake of computation, this reconstruction is directly operated on the reference configuration by means of a harmonic extension of the structure displacement $\widehat{\mathbf{d}}_{s}$ at the fluid-structure interface $\widehat{\Gamma}$ to the interior of the reference fluid domain $\widehat{\Omega}_{f}$, i.e.,

$$
\left\{\begin{aligned}
-\Delta \widehat{\mathbf{d}}_{f} & =0 & & \text { in } \widehat{\Omega} \\
\widehat{\mathbf{d}}_{f} & =\widehat{\mathbf{d}}_{s} & & \text { on } \widehat{\Gamma} .
\end{aligned}\right.
$$

Other types of extensions are also possible; for example one may use the linear elasticity equations $[15,110]$. In our experience, this is necessary in presence of large (but smooth) boundary displacements. As the solid displacement $\widehat{\mathbf{d}}_{s}$ changes in time, equation (2.2a) allows to define the current fluid domain configuration $\Omega_{f}(t)=\mathcal{A}_{t}\left(\widehat{\Omega}_{f}\right)$ thanks to the ALE map parametrization (2.1).

In our FSI model, the fluid dynamics is governed by the incompressible NavierStokes equations written in the ALE frame of reference [15, 45, 65, 37], for almost all $t \in(0, T)$,

$$
\left\{\begin{aligned}
\left.\rho_{f} \frac{\partial \mathbf{u}}{\partial t}\right|_{\mathcal{A}_{t}}+\rho_{f}((\mathbf{u}-\mathbf{w}) \cdot \nabla) \mathbf{u}-\nabla \cdot \boldsymbol{\sigma}_{f} & =0 & & \text { in } \Omega_{f}(t), \\
\nabla \cdot \mathbf{u} & =0 & & \text { in } \Omega_{f}(t), \\
\mathbf{u} & =\mathbf{h}_{f} & & \text { on } \Gamma_{D}^{f}, \\
\boldsymbol{\sigma}_{f} \mathbf{n}_{f} & =\mathbf{g}_{f} & & \text { on } \Gamma_{N}^{f}, \\
\mathbf{u} \circ \mathcal{A}_{t} & =\frac{\partial \widehat{\mathbf{d}}_{s}}{\partial t} & & \text { on } \hat{\Gamma},
\end{aligned}\right.
$$

where $\left.\frac{\partial}{\partial t}\right|_{\mathcal{A}_{t}}=\frac{\partial}{\partial t}+\mathbf{w} \cdot \nabla$ is the ALE derivative, $\mathbf{w}=\frac{\partial \mathcal{A}_{t}}{\partial t} \circ \mathcal{A}_{t}^{-1}$ is fluid domain velocity, $\mathbf{u}$ and $p$ are the velocity and pressure of the fluid, respectively, and $\mathbf{n}_{f}$ the outward unit normal vector to $\partial \Omega_{f}(t)$. We denoted by $\rho_{f}$ the density of the fluid and by $\boldsymbol{\sigma}_{f}$ the Cauchy stress tensor

$$
\boldsymbol{\sigma}_{f}=\mu_{f}\left(\nabla \mathbf{u}+(\nabla \mathbf{u})^{T}\right)-p \mathbf{I},
$$


with $\mathbf{I}$ being the identity tensor, $\mu_{f}$ the dynamic viscosity of the fluid. The functions $\mathbf{h}_{f}$ and $\mathbf{g}_{f}$ indicate the Dirichlet and Neumann data applied at the the Dirichlet and Neumann boundaries $\Gamma_{D}^{f}$ and $\Gamma_{N}^{f}$, respectively, of $\Omega_{f}(t)$; equation 2.3 a represents the continuity of the velocities at the interface.

We formulate the full three-dimensional structure problem in a Lagrangian frame of reference. The conservation of momentum for the structure reads

$$
\left\{\begin{aligned}
\widehat{\rho}_{s} \frac{\partial^{2} \hat{\mathbf{d}}_{s}}{\partial t^{2}}-\nabla_{\widehat{\mathbf{x}}} \cdot \widehat{\mathbf{\Pi}}\left(\widehat{\mathbf{d}}_{s}\right) & =0 & & \text { in } \widehat{\Omega}_{s} \times(0, T], \\
\widehat{\mathbf{d}}_{s} & =\mathbf{h}_{s} & & \text { on } \widehat{\Gamma}_{D}^{s} \times(0, T], \\
\widehat{\mathbf{\Pi}}\left(\widehat{\mathbf{d}}_{s}\right) \widehat{\mathbf{n}}_{s} & =0 & & \text { on } \widehat{\Gamma}_{N}^{s} \times(0, T], \\
(\operatorname{det}[\mathbf{F}]) \widehat{\mathbf{\Pi}}\left(\widehat{\mathbf{d}}_{s}\right) \mathbf{F}^{T} \widehat{\mathbf{n}}_{s}+\boldsymbol{\sigma}_{f} \mathbf{n}_{f} & =0 & & \text { on } \Gamma \times(0, T] .
\end{aligned}\right.
$$

Here $\widehat{\mathbf{n}}_{s}$ represent the outward unit normal vector to $\partial \widehat{\Omega}_{s}$ and $\mathbf{F}=\mathbf{I}+\nabla_{\hat{\mathbf{x}}} \hat{\mathbf{d}}_{s}$ is the deformation gradient tensor. The function $\mathbf{h}_{s}$ indicates the Dirichlet data applied at the Dirichlet boundary $\Gamma_{D}^{s}$ of $\widehat{\Omega}_{s}$. The material is characterized by a density $\widehat{\rho}_{s}$, Young's modulus $E_{s}$ and the Poisson's ratio $\nu_{s}$, which define in turn the first Piola-Kirchhoff stress tensor

$$
\widehat{\mathbf{\Pi}}\left(\widehat{\mathbf{d}}_{s}\right)=\lambda_{s} \operatorname{tr}\left(\frac{\nabla_{\widehat{\mathbf{x}}} \hat{\mathbf{d}}_{s}+\left(\nabla_{\widehat{\mathbf{x}}} \hat{\mathbf{d}}_{s}\right)^{T}}{2}\right) \mathbf{I}+\mu_{s}\left(\nabla_{\widehat{\mathbf{x}}} \widehat{\mathbf{d}}_{s}+\left(\nabla_{\widehat{\mathbf{x}}} \hat{\mathbf{d}}_{s}\right)^{T}\right),
$$

where

$$
\lambda_{s}=\frac{E_{s} \nu_{s}}{\left(1-2 \nu_{s}\right)\left(1+\nu_{s}\right)} \quad \text { and } \quad \mu_{s}=\frac{E_{s}}{2\left(1+\nu_{s}\right)}
$$

are the first and second Lamé coefficients, respectively. Other constitutive laws are of course also possible, here we choose the simplest one.

The coupling between these three subproblems is ensured by imposing the geometry adherence, the continuity of the velocity and the continuity of the normal component of the stresses at the interface through Equations (2.2b), (2.3a), and (2.4b), respectively. Defining the physical sub-problems with (2.3a) and (2.4b) is arbitrary, one could choose to swap them. We notice that the resulting coupled system is nonlinear due to the convective term in the fluid momentum equation, to the possible use of nonlinear material laws to model the structure deformation and to the moving computational fluid domain.

\subsection{Weak formulation}

We recall the weak form of the FSI problem in nonconservative form. The velocity coupling condition is imposed strongly, while the continuity of the normal component 
of the stresses is imposed in weak form. We presume that $\mathcal{A}_{t}$ is a diffeomorphism for ant time $t \in[0, T]$ and introduce introduce the following functional spaces:

$$
\begin{aligned}
U^{f} & =\left\{\mathbf{v}=\widehat{\mathbf{v}} \circ \mathcal{A}_{t}^{-1} \mid \widehat{\mathbf{v}} \in\left[H^{1}\left(\widehat{\Omega}_{f}\right)\right]^{3}\right\}, \\
U_{D}^{f} & =\left\{\mathbf{v}=\in U^{f}, \mathbf{v}=0 \text { on } \Gamma_{D}^{f}(t)\right\}, \\
Q^{f} & =\left\{q=\widehat{q} \circ \mathcal{A}_{t}^{-1} \mid \widehat{q} \in L^{2}\left(\widehat{\Omega}_{f}\right)\right\}, \\
U^{s} & =\left[H^{1}\left(\widehat{\Omega}_{s}\right)\right]^{3}, U_{D}^{s}=\left\{\mathbf{v} \in U^{s} \mid \mathbf{v}=0 \text { on } \Gamma_{D}^{s}\right\}, \\
U^{g} & =\left[H^{1}\left(\widehat{\Omega}_{f}\right)\right]^{3}, U_{D}^{g}=\left\{\mathbf{v} \in U^{g} \mid \mathbf{v}=0 \text { on } \Gamma_{\text {fixed }}^{f}\right\}, \\
U^{\lambda} & =\left[H^{1 / 2}(\widehat{\Gamma})\right]^{3} .
\end{aligned}
$$

$\Gamma_{\text {fixed }}^{f}$ is the part of the fluid boundary $\widehat{\Omega}_{f}$ where the domain motion is prescribed (e.g. when a portion of the fluid domain boundary is fixed). The weak form of the fluid and structure equations is standard [45]. We introduce an auxiliary variable $\lambda \in U^{\lambda}$ representing the normal stresses exerted by the fluid on the interface $\Gamma$ and which, thanks to $(2.4 \mathrm{~b})$, can be written as:

$\boldsymbol{\lambda} \in U^{\lambda}: \int_{\hat{\Gamma}} \boldsymbol{\lambda} \cdot \boldsymbol{\eta} d \gamma=\int_{\Gamma(t)} \sigma_{f} \mathbf{n}_{f} \cdot \boldsymbol{\eta} \circ \mathcal{A}_{t}^{-1} d \gamma=-\int_{\hat{\Gamma}} \Pi\left(\widehat{\mathbf{d}}_{s}\right) \widehat{\mathbf{n}}_{s} \cdot \boldsymbol{\eta} d \hat{\gamma} \quad \forall \boldsymbol{\eta} \in U^{\lambda}$.

We recall the notation for the Dirichlet boundary data for the fluid, structure, and geometry sub-problem: $\mathbf{h}_{f}: \Gamma_{D}^{f} \rightarrow \mathbb{R}^{2}, \mathbf{h}_{s}: \Gamma_{D}^{s} \rightarrow \mathbb{R}^{2}, \mathbf{h}_{g}: \Gamma_{\text {fixed }}^{f} \rightarrow \mathbb{R}^{2}$, respectively. The weak form of the FSI problem reads: for all $t \in(0, T]$, find $\left(\mathbf{u}, p, \widehat{\mathbf{d}}_{s}, \widehat{\mathbf{d}}_{f}, \boldsymbol{\lambda}\right) \in$ $U^{f} \times Q^{f} \times U^{s} \times U^{g} \times U^{\lambda}$ such that $\mathbf{u}=\mathbf{h}_{f}$ on $\Gamma_{D}^{f}, \widehat{\mathbf{d}}_{s}=\mathbf{h}_{s}$ on $\Gamma_{D}^{s}, \widehat{\mathbf{d}}_{f}=\mathbf{h}_{g}$ on $\Gamma_{\text {fixed }}^{f}$, satisfying

$$
\begin{array}{ll}
\int_{\Omega_{f}(t)} & \left(\left.\rho_{f} \frac{\partial \mathbf{u}}{\partial t}\right|_{\mathcal{A}_{t}} \cdot \mathbf{v}_{f}+\rho_{f}((\mathbf{u}-\mathbf{w}) \cdot \nabla) \mathbf{u} \cdot \mathbf{v}_{f}+\boldsymbol{\sigma}_{f}: \nabla \mathbf{v}_{f}\right) d \Omega \\
+\quad & \int_{\widehat{\Gamma}} \boldsymbol{\lambda} \cdot\left(\mathbf{v}_{f} \circ \mathcal{A}_{t}\right) d \hat{\gamma}=\int_{\Gamma_{N}^{f}} \mathbf{g}_{f} \cdot \mathbf{v}_{f} d \gamma \\
\int_{\Omega_{f}(t)} & q \nabla \cdot \mathbf{u} d \Omega=0 \\
\int_{\widehat{\Omega}_{s}} & \left(\widehat{\rho}_{s} \frac{\partial^{2} \widehat{\mathbf{d}}_{s}}{\partial t^{2}} \cdot \mathbf{v}_{s}+\widehat{\Pi}\left(\widehat{\mathbf{d}}_{s}\right): \nabla_{\hat{\mathbf{x}}} \mathbf{v}_{s}\right) d \Omega-\int_{\widehat{\Gamma}} \boldsymbol{\lambda} \cdot \mathbf{v}_{s} d \hat{\gamma}=\int_{\Gamma_{N}^{s}} \mathbf{g}_{s} \cdot \mathbf{v}_{s} d \gamma \\
\int_{\widehat{\Gamma}} \quad\left(\mathbf{u}_{f} \circ \mathcal{A}_{t}\right) \cdot \boldsymbol{\eta} d \gamma-\int_{\widehat{\Gamma}} \frac{\partial \widehat{\mathbf{d}}_{s}}{\partial t} \cdot \boldsymbol{\eta} d \gamma=0 \\
\int_{\widehat{\Gamma} \quad} \quad \nabla_{\hat{\mathbf{x}}} \hat{\mathbf{d}}_{f}: \nabla_{\hat{\mathbf{x}}} \mathbf{v}_{g} d \Omega=0 \quad \forall\left(\mathbf{v}_{f}, q, \mathbf{v}_{s}, \mathbf{v}_{g}, \boldsymbol{\eta}\right) \in U_{D}^{f} \times Q^{f} \times U_{D}^{s} \times U_{D}^{g} \times U^{\lambda} \\
\widehat{\mathbf{d}}_{f} \quad=\widehat{\mathbf{d}}_{s} \quad \text { on } \widehat{\Gamma} .
\end{array}
$$


Note that $\boldsymbol{\eta}$ represents the Lagrange multiplier which enforces the equilibrium of the stresses $(2.4 \mathrm{~b})$ in weak form. From the variational viewpoint, the normal stresses are in $H^{-1 / 2}(\Gamma)$, it is therefore natural to impose their equilibrium only weakly. A strong treatment would not be consistent. From (2.6) and (2.7) we observe that

$$
\int_{\Gamma(t)} \sigma_{f} \mathbf{n}_{f} \cdot \boldsymbol{\eta} \circ \mathcal{A}_{t}^{-1} d \gamma+\int_{\hat{\Gamma}} \Pi\left(\widehat{\mathbf{d}}_{s}\right) \widehat{\mathbf{n}}_{s} \cdot \boldsymbol{\eta} d \hat{\gamma}=0 \quad \forall \boldsymbol{\eta} \in U^{\lambda}
$$

\subsection{Fully implicit monolithic scheme}

A large variety of different time discretization schemes for the coupled set of equations describing the FSI phenomena can be found in literature. One approach may consist in dealing with all the nonlinearities of the problem in a fully implicit fashion, leading to the so-called fully implicit strategy $[41,59,14,49,127]$. Although this approach is the most stable, it is also the most expensive. Alternative time discretizations, depending on the targeted application, can be devised. For instance, in the field of hemodynamics, a Convective Explicit (CE) time discretization is used in [27], in which the nonlinear convective term in the unsteady Navier-Stokes equations is linearized by means of extrapolation. Further, a Geometry-Convective Explicit (GCE) discretization is proposed in [7] wherein the moving geometry is taken at the previous time step and the convective term is treated semi-implicitly, thus leading to a linear problem to be solved at each discrete time step (provided that a linear material law is used for the solid).

We consider a Fully Implicit (FI) scheme for which all the nonlinearities are treated implicitly. In space, we consider a Galerkin finite elements approximation. Stabilized P1-P1 elements are used to approximate the fluid velocity and pressure variables with VMS-SUPG stabilization [13,46]. The solid displacement and ALE fields are discretized by $\mathrm{P} 1$ finite elements. When considering the reduced model presented in section 3, we use Taylor-Hood P2-P1 finite elements without stabilization for the fluid, while the structure is integrated into the fluid variable.

For the sake of brevity, here we do not report here the finite element formulation since it differ from (2.7) only on the spaces. When using the VMS-SUPG stabilization, the following terms are added to the Galerkin formulation. Inside each element $K$ of the fluid tetrahedral mesh $\mathcal{T}_{f}^{h}$ representing the current fluid domain $\Omega_{f}(t)$, the finite element solution is a linear function and it is possible to compute the strong residual of the momentum equation as

$$
\left.\mathbf{r}_{K}(\mathbf{u}, p)=\left.\rho_{f} \frac{\partial \mathbf{u}}{\partial t}\right|_{\mathcal{A}_{t}}+\rho_{f}((\mathbf{u}-\mathbf{w}) \cdot \nabla) \mathbf{u}+\nabla p-\mu \Delta \mathbf{u}\right)
$$


The VMS-SUPG stabilising terms are then computed as

$$
\begin{aligned}
& \sum_{K \in \mathcal{T}_{f}^{h}} \int_{K}\left((\mathbf{u}-\mathbf{w}) \cdot \nabla \mathbf{v}_{f}+q\right) \tau_{m} \mathbf{r}_{K}(\mathbf{u}, p) d \Omega \\
+ & \sum_{K \in \mathcal{T}_{f}^{h}} \int_{K} \nabla \cdot \mathbf{v}_{f} \tau_{c} \nabla \cdot \mathbf{u} d \Omega \\
+ & \sum_{K \in \mathcal{T}_{f}^{h}} \int_{K}(\mathbf{u}-\mathbf{w}) \cdot\left(\nabla \mathbf{v}_{f}\right)^{T} \tau_{m} \mathbf{r}_{K}(\mathbf{u}, p) d \Omega,
\end{aligned}
$$

where the first two rows represent classical Streamline-Upwind Petrov-Galerkin (SUPG) stabilization terms and the third row stems from the Variational Multiscale (VMS) technique applied to the Navier-Stokes equations. In the stabilization terms we denoted by $K \in \mathcal{T}_{f}^{h}$ one tetrahedron of the fluid mesh, while $\tau_{m}$ and $\tau_{c}$ are two stabilization parameters defined as:

$$
\begin{aligned}
\tau_{m} & =\frac{1}{\sqrt{\frac{4 \rho_{f}^{2}}{\Delta t^{2}}+\rho_{f}^{2}(\mathbf{u}-\mathbf{w}) \cdot \mathbf{G}(\mathbf{u}-\mathbf{w})+C_{r} \mu_{f}^{2} \mathbf{G}: \mathbf{G}}}, \\
\tau_{c} & =\left(\tau_{m} \mathbf{g} \cdot \mathbf{g}\right)^{-1} .
\end{aligned}
$$

In equations (2.9) and (2.10), the covariant metric tensor $\mathbf{G}$ is defined componentwise as $G_{i, j}=\sum_{k=1}^{3} \frac{\partial \eta_{k}}{\partial x_{i}} \frac{\partial \eta_{k}}{\partial x_{i}}$ and is related to the geometrical mapping with $\boldsymbol{\eta}=$ $\left(\eta_{1}, \eta_{2}, \eta_{3}\right)$, while the vector $\mathbf{g}$ has components $g_{i}=\sum_{k=1}^{3} \frac{\partial \eta_{k}}{\partial x_{i}}$. The constant $C_{r}>0$ depends on the degree $p$ of the basis functions and we set it as an element-wise inverse estimate $C_{r}=60 \cdot 2^{p-2}$ as suggested by [13].

We discretize the time derivative of the fluid problem by second order backward differentation formulas $[54,46]$

$$
\left.\frac{\partial \mathbf{u}}{\partial t}\left(t^{n+1}\right)\right|_{\mathcal{A}_{t}} \approx \frac{3 \mathbf{u}^{n+1}-4 \mathbf{u}^{n}+\mathbf{u}^{n-1}}{2 \Delta t}
$$

where $\Delta t$ is the time step size, $\left\{t^{n}\right\}_{n=1,2, \ldots}$ are the times at which we solve the discrete space problem and $\mathbf{u}^{n+1}$ is the approximation of $\mathbf{u}$ at time $t^{n+1}$. The time discretization of the structural problem is carried out by the Newmark method [91]

$$
\frac{\partial^{2} \widehat{\mathbf{d}}_{s}}{\partial t^{2}}\left(t^{n+1}\right) \approx \frac{1}{\beta \Delta t^{2}} \widehat{\mathbf{d}}_{s}^{n+1}-\frac{1}{\beta \Delta t^{2}}\left(\widehat{\mathbf{d}}_{s}^{n}+\Delta t \dot{\hat{\mathbf{d}}}_{s}^{n}\right)-\frac{1-2 \beta}{2 \beta} \ddot{\ddot{\mathbf{d}}}_{s},
$$


where $\beta=0.25, \gamma=0.5$ and:

$$
\begin{aligned}
& \ddot{\hat{\mathbf{d}}}_{s}^{n}=\frac{1}{\beta \Delta t^{2}} \hat{\mathbf{d}}_{s}^{n}-\frac{1}{\beta \Delta t^{2}}\left(\widehat{\mathbf{d}}_{s}^{n-1}+\Delta t \dot{\hat{\mathbf{d}}}_{s}^{n-1}\right)-\frac{1-2 \beta}{2 \beta} \ddot{\mathbf{d}}_{s}^{n-1}, \\
& \dot{\hat{\mathbf{d}}}_{s}^{n}=\dot{\hat{\mathbf{d}}}_{s}^{n-1}+\Delta t\left(\gamma \ddot{\hat{\mathbf{d}}}_{s}^{n}+(1-\gamma) \ddot{\hat{\mathbf{d}}}_{s}^{n-1}\right) .
\end{aligned}
$$

At each time step, the resulting nonlinear system to be solved may be rewritten as

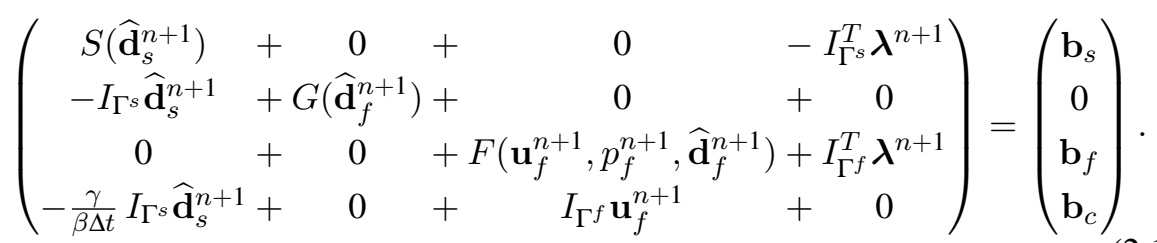

We denoted by $\widehat{\mathbf{d}}_{s}^{n+1}, \mathbf{d}^{n+1}, \mathbf{u}^{n+1}, p^{n+1}$, and $\boldsymbol{\lambda}^{n+1}$, the unknown displacement of the structure, the displacement of the fluid domain, the velocity and pressure of the fluid, and the Lagrange multipliers, respectively. We make use of an augmented formulation wherein the Lagrange multipliers are used to impose the continuity of the velocity at the fluid-structure interface.

The diagonal blocks on the left hand side of (2.15) account for the discretized solid, geometry and fluid problems. We remark that $F$ is nonlinear due to the convective term and the fact that the fluid domain moves. The matrices $I_{\Gamma^{f}}$ and $I_{\Gamma^{s}}$ are the restriction of fluid velocity and structure displacement vectors to the interface and in (2.15) account for the continuity of velocities and the geometry adherence, which are imposed strongly. Their transposes account for the continuity of the normal component of the stresses, which is imposed weakly. Last row of (2.15) represents the discretized kinematic coupling condition at the fluid-structure interface:

$$
I_{\Gamma^{f}} \mathbf{u}^{n+1}-\frac{\gamma}{\beta \Delta t} I_{\Gamma^{s}} \widehat{\mathbf{d}}_{s}^{n+1}=\mathbf{b}_{c}
$$

where

$$
\mathbf{b}_{c}=I_{\Gamma^{s}} \dot{\hat{\mathbf{d}}}_{s}^{n}-\frac{\gamma}{\beta \Delta t}\left(I_{\Gamma^{s}} \widehat{\mathbf{d}}_{s}^{n}+\Delta t I_{\Gamma^{s}} \dot{\hat{\mathbf{d}}}_{s}^{n}\right)-\Delta t \gamma \frac{1-2 \beta}{2 \beta} I_{\Gamma^{s}} \ddot{\widehat{\mathbf{d}}}_{s}^{n}+\Delta t(1-\gamma) I_{\Gamma^{s}} \ddot{\widehat{\mathbf{d}}}_{s}^{n} .
$$

We assumed that the fluid and structural meshes, as well as the polynomial degree are conforming at the interface. To account for non-conforming fluid-structure interface discretizations, please refer to [87, 47].

\subsection{Algebraic formulation}

We solve the nonlinear fully coupled FSI problem (2.15) using the Newton method as in, e.g., $[41,59,14]$. The solution of (2.15) at time $t^{n}=n \Delta t$ is denoted by $\mathbf{X}^{n}=\left(\widehat{\mathbf{d}}_{s}^{n}, \widehat{\mathbf{d}}_{f}^{n}, \mathbf{u}^{n}, p^{n}, \boldsymbol{\lambda}^{n}\right)^{T}$. At each time step, we first extrapolate an approximate 
solution $\mathbf{X}_{0}^{n+1}$ from the previous time levels by Newton Gregory backward polynomials. In particular, since we use second order time integrators, we set $\mathbf{X}_{0}^{n+1}=2 \mathbf{X}^{n}-$ $\mathbf{X}^{n-1}$. Then, we compute a sequence of approximations $\mathbf{X}_{1}^{n+1}, \mathbf{X}_{2}^{n+1}$, etc. until the numerical solution converges up to a prescribed tolerance. The generic $k+1$ iteration of the Newton method applied to (2.15) is described as follows. Starting from an approximation of $\mathbf{X}_{k}^{n+1}$, we compute the residual $\mathbf{R}_{k}^{n+1}=\left(\mathbf{r}_{\mathbf{d}_{s}, k}^{n+1}, \mathbf{r}_{\mathbf{d}_{f}, k}^{n+1}, \mathbf{r}_{u, k}^{n+1}, r_{p, k}^{n+1}, \mathbf{r}_{\lambda, k}^{n+1}\right)^{T}$ :

$$
\mathbf{R}_{k}^{n+1}=\left(\begin{array}{c}
\mathbf{b}_{s} \\
0 \\
\mathbf{b}_{f} \\
\mathbf{b}_{c}
\end{array}\right)-\left(\begin{array}{cc}
S\left(\widehat{\mathbf{d}}_{s, k}^{n+1}\right) & -I_{\Gamma^{s}}^{T} \boldsymbol{\lambda}_{k}^{n+1} \\
-I_{\Gamma^{s}} \widehat{\mathbf{d}}_{s, k}^{n+1} & +G\left(\widehat{\mathbf{d}}_{f, k}^{n+1}\right) \\
F\left(\mathbf{u}_{k}^{n+1}, p_{k}^{n+1}, \widehat{\mathbf{d}}_{f, k}^{n+1}\right) & +I_{\Gamma^{f}}^{T} \boldsymbol{\lambda}_{k}^{n+1} \\
-\frac{\gamma}{\beta \Delta t} I_{\Gamma^{s}} \widehat{\mathbf{d}}_{s, k}^{n+1} & +I_{\Gamma^{f}} \mathbf{u}_{k}^{n+1}
\end{array}\right) .
$$

Then, we compute the Newton correction vector $\delta \mathbf{X}_{k}^{n+1}=\left(\delta \widehat{\mathbf{d}}_{s, k}^{n+1}, \delta \widehat{\mathbf{d}}_{f, k}^{n+1}, \delta \mathbf{u}_{k}^{n+1}, \delta p_{k}^{n+1}\right.$, $\left.\delta \boldsymbol{\lambda}_{k}^{n+1}\right)^{T}$ by solving the Jacobian linear system

$$
J_{F S I} \delta \mathbf{X}_{k}^{n+1}=-\mathbf{R}_{k}^{n+1},
$$

being

$$
J_{F S I}=\left(\begin{array}{cccc}
\mathcal{S} & 0 & 0 & -I_{\Gamma^{s}}^{T} \\
-I_{\Gamma^{s}} & \mathcal{G} & 0 & 0 \\
0 & \mathcal{D} & \mathcal{F} & I_{\Gamma^{f}}^{T} \\
-\frac{\gamma}{\beta \Delta t} I_{\Gamma^{s}} & 0 & I_{\Gamma^{f}} & 0
\end{array}\right),
$$

where $\mathcal{S}, \mathcal{G}$ and $\mathcal{F}$ represent the linearized structure, geometry and fluid problems, respectively; $\mathcal{D}$ are the shape derivatives, i.e. the derivatives of $F\left(\mathbf{u}^{n+1}, p^{n+1}, \widehat{\mathbf{d}}_{f}^{n+1}\right)$ with respect to $\widehat{\mathbf{d}}_{f}^{n+1}$ (see [41] for their exact computation).

Finally, we update the solution: $\mathbf{X}_{k+1}^{n+1}=\mathbf{X}_{k}^{n+1}+\delta \mathbf{X}_{k}^{n+1}$. The Newton iterations are stopped when

$$
\frac{\left\|\mathbf{R}_{k}^{n+1}\right\|_{\infty}}{\left\|\mathbf{R}_{0}^{n+1}\right\|_{\infty}} \leq \epsilon
$$

where $\mathbf{R}_{0}^{n+1}$ is the residual at the first Newton iteration and $\epsilon$ is a given tolerance.

\subsection{Preconditioning by FaCSI}

At each iteration of the Newton method, linear system (2.19) is solved by the GMRES method preconditioned by FaCSI [32]. FaCSI exploits the factorized form of the FSI Jacobian matrix, the use of static condensation to formally eliminate the interface degrees of freedom of the fluid equations, and the use of SIMPLE for the condensed fluid system. The FaCSI preconditioner reads:

$$
P_{F a C S I}=P_{\mathcal{S}}^{a p} \cdot P_{\mathcal{G}}^{a p} \cdot P_{\mathcal{F}}^{a p},
$$


where:

$$
P_{\mathcal{S}}^{a p}=\left(\begin{array}{cccc}
\mathcal{H}_{\mathcal{S}} & 0 & 0 & 0 \\
0 & I & 0 & 0 \\
0 & 0 & I & 0 \\
0 & 0 & 0 & I
\end{array}\right), P_{\mathcal{G}}^{a p}=\left(\begin{array}{cccc}
I & 0 & 0 & 0 \\
-I_{\Gamma^{s}} & \mathcal{H}_{\mathcal{G}} & 0 & 0 \\
0 & 0 & I & 0 \\
0 & 0 & 0 & I
\end{array}\right), P_{\mathcal{F}}^{a p}=\left(\begin{array}{cccc}
I & 0 & 0 & 0 \\
0 & I & 0 & 0 \\
0 & \mathcal{D} & \\
-\frac{\gamma}{\beta \Delta t} I_{\Gamma^{s}} & 0 &
\end{array}\right)
$$

In the preconditioner we have dropped the off-diagonal block $-I_{\Gamma^{s}}^{T}$ of $J_{F S I}$. In the application of FaCSI, the inverses of the diagonal blocks associated with the structure and geometry problems are approximated by efficient preconditioners denoted by $\mathcal{H}_{\mathcal{S}}$ (structure problem) and $\mathcal{H}_{\mathcal{G}}$ (geometry problem). The preconditioner for the fluid and Lagrange multiplier parts, denoted by $\mathcal{H}_{\mathcal{F}}$, is built upon static condensation to formally eliminate the interface degrees of freedom of the fluid equations and the use of SIMPLE preconditioner for the unsteady Navier-Stokes equations.

For the sake of clarity, below we report how the preconditioner of the fluid problem $P_{\mathcal{F}}^{a p}$ applies to a given linear residual $\mathbf{r}=\left(\mathbf{r}_{\mathbf{d}_{s}}, \mathbf{r}_{\mathbf{d}_{f}}, \mathbf{r}_{\mathbf{u}}, \mathbf{r}_{p}, \mathbf{r}_{\boldsymbol{\lambda}}\right)^{T}$ :

(i) compute $\mathbf{z}_{F}=\left(\mathbf{r}_{\mathbf{u}}, \mathbf{r}_{p}\right)^{T}-\mathcal{D} \mathbf{r}_{\mathbf{d}_{f}}$ and $\mathbf{z}_{\boldsymbol{\lambda}}=\mathbf{r}_{\lambda}+\frac{\gamma}{\beta \Delta t} I_{\Gamma^{s}} \mathbf{r}_{\mathbf{d}_{s}}$

(ii) after denoting by $\mathbf{w}_{\mathbf{u}}$ and $\mathbf{w}_{p}, \mathbf{z}_{\mathbf{u}}$ and $\mathbf{z}_{p}$ the velocity and pressure components of $\mathbf{w}_{F}$ and $\mathbf{z}_{F}$, respectively, we set $\mathbf{z}_{\mathbf{u}, \Gamma}=\mathbf{z}_{\boldsymbol{\lambda}}$

(iii) application of the SIMPLE preconditioner, which involves the following steps:

a) $\mathbf{y}_{\mathbf{u}, i}=\mathcal{H}_{\mathcal{K}_{i i}}^{-1}\left(\mathbf{z}_{\mathbf{u}, i}-\mathcal{K}_{i \Gamma} \mathbf{z}_{\mathbf{u}, \Gamma}\right)$,

b) $\mathbf{w}_{p}=\mathcal{H}_{\widetilde{S}}^{-1}\left(\mathcal{B}_{i} \mathbf{y}_{\mathbf{u}, i}-\mathbf{z}_{p}+\mathcal{B}_{\Gamma} \mathbf{z}_{\mathbf{u}, \Gamma}\right)$,

c) $\mathbf{w}_{\mathbf{u}}=\left(\mathbf{w}_{\mathbf{u}, i}, \mathbf{w}_{\mathbf{u}, \Gamma}\right)^{T}=\left(\mathbf{y}_{\mathbf{u}, i}-D^{-1} \mathcal{B}_{i}^{T} \mathbf{w}_{p}, \mathbf{z}_{\mathbf{u}, \Gamma}\right)^{T}$.

Finally, we compute $\mathbf{w}_{\boldsymbol{\lambda}}=I_{\Gamma^{u}}\left(\mathbf{z}_{\mathbf{u}}-\mathcal{K} \mathbf{w}_{\mathbf{u}}-\mathcal{B}^{T} \mathbf{w}_{p}\right)$.

In the application of the SIMPLE preconditioner we denoted by $\mathcal{K}$ and $\mathcal{B}$ the matrices representing the linearized advection-diffusion-reaction and gradient terms of the fluid problem split into their internal (index $i$ ) and interface (index $\Gamma$ ) components. The matrix $D$ is the diagonal of $\mathcal{K}_{i i}, \mathcal{H}_{\mathcal{K}_{i i}}$ is the preconditioner associated with $\mathcal{K}_{i i}$ and $\mathcal{H}_{\widetilde{S}}$ is an approximation of the Schur complement $\mathcal{B}_{i} D^{-1} \mathcal{B}_{i}^{T}$ of the fluid problem.

In this work we make use of the one level Algebraic Additive Schwarz (AAS) method for $\mathcal{H}_{\mathcal{S}}, \mathcal{H}_{\mathcal{G}}$, while the 3 level Alebraic Multigrid (AMG) method is used for $\mathcal{H}_{\mathcal{K}_{i i}}$ and $\mathcal{H}_{\widetilde{S}}$. We remark that the exact local subdomain solves for AAS as well as the exact coarse solve of the AMG preconditioner are carried out by LU factorization using the library MUMPS [2, 3].

\section{Modeling reduction for FSI in arteries}

In Section 2 we focused on parallel algorithms to solve the three dimensional FSI problems. Now we make a trend reversal and we proceed with a complexity reduction 
of the FSI system. In particular, our goal is to compute an approximation of specific target outputs using a small amount of computational resources. . As we said in the introduction, this challenge can be faced exploiting reduced order models that are meant for approximating the solution of parametric PDE system in real time. .

Applying numerical reduction to fully three dimensional realistic FSI problems may be even more challenging than solving FSI problems themselves. To begin with, we start by introducing further modelling assumptions which aims at reducing the model complexity of the original 3D nonlinear FSI system.

Once the model reduction has been completed, it is possible to proceed with its numerical reduction by Reduced Order Modeling (ROM) based on Proper Orthogonal Decomposition (POD).

\subsection{Transpiration conditions}

First, we focus on the complexity due to the motion of the fluid domain. We make use of transpiration conditions [31,40] with the specific goal of formulating the FSI problem on a fixed computational domain. Transpiration conditions are based on a Taylor expansion of the system variables. For instance, the Taylor expansion of the velocity $\mathbf{u}$ at a point of the current configuration $\mathbf{x}=\mathcal{A}_{t}(\widehat{\mathbf{x}})$ in the neighborhood of the point $\widehat{\mathbf{x}}$ (see Figure 1) reads:

$$
\mathbf{u}\left(\mathcal{A}_{t}(\widehat{\mathbf{x}})\right)=\mathbf{u}(\widehat{\mathbf{x}})+\left.\nabla_{\widehat{\mathbf{x}}} \mathbf{u}\right|_{\widehat{\mathbf{x}}} \widehat{\mathbf{d}_{s}}+O\left(\left\|\left.\widehat{\mathbf{d}_{s}}\right|_{\widehat{\Gamma}}\right\|^{2}\right),
$$

where (3.1) is a first order approximation. Furtherly, if we truncate the expansion at the zeroth order, we obtain:

$$
\mathbf{u}\left(\mathcal{A}_{t}(\widehat{\mathbf{x}})\right)=\mathbf{u}(\widehat{\mathbf{x}})+O\left(\left\|\widehat{\mathbf{d}_{s}} \mid \widehat{\Gamma}\right\|\right) .
$$

In equations (3.1) and (3.2) we used the fact that on the interface $\widehat{\Gamma}$ the following identity holds:

$$
\widehat{\mathbf{d}}_{s}=\mathcal{A}_{t}(\widehat{\mathbf{x}})-\widehat{\mathbf{x}}
$$

The Taylor expansion allows us to write the velocity $\mathbf{u}$ as a function of $\widehat{\mathbf{x}}$ on the reference fixed configuration $\widehat{\Omega}_{f}$ which usually corresponds to the initial configuration $\Omega_{f}(0)$. There is a crucial difference between choosing transpiration conditions of first or zeroth order. Indeed, if we choose first order transpiration conditions, on one side, we would manage to fix the computational domain, on the other side, we would introduce several non-linear terms $\left(\left.\nabla_{\widehat{\mathbf{x}}} \mathbf{u}\right|_{\widehat{\mathbf{x}}} \widehat{\mathbf{d}}_{s}\right)$. We opt then for zeroth order expansions, although this may affect the results with a modelling error of the same order as that one of the interface displacement.

Since transpiration conditions allow us to approximate the current configuration with the reference one, there is no need to differentiate the notation. In what follows we simplify the notation removing the $\widehat{\cdot}$, e.g.:

$$
\Omega_{f}(t) \approx \widehat{\Omega}_{f}=: \Omega_{f} \quad \text { and } \quad \Gamma(t) \approx \widehat{\Gamma}=: \Gamma
$$




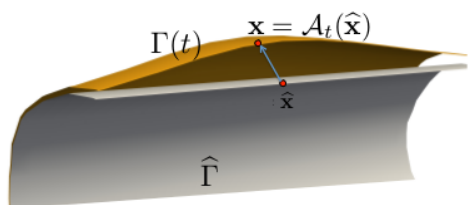

Figure 1: Graphical representation of the transpiration conditions

The strong formulation of the FSI problem wherein tranpiration conditions are used can be derived from system (2.7) applying the following three modifications:

- the domain $\Omega_{f}(t)$ is substituted in the domain $\Omega_{f}$;

- the ALE velocity w disappears since it is identically equal to zero;

- the kinematic coupling condition (2.3a) can be rewritten as follows:

$$
\frac{\partial \mathbf{d}_{s}(\mathbf{x})}{\partial t}=\mathbf{u}(\mathbf{x}) \quad \text { for } \quad \mathbf{x} \in \Gamma .
$$

Finally, we remark that an equivalent Taylor expansion as the one written for the velocity $\mathbf{u}$ can be performed on the fluid Cauchy tensor $\sigma_{f}$, thus approximating $\sigma_{f}(\mathbf{x})$ with $\sigma_{f}(\widehat{\mathbf{x}})$. At this point, also the dynamic coupling condition (2.8) can be reformulated and reads as follows:

$$
\int_{\Gamma} \sigma_{f} \mathbf{n}_{f} \cdot \boldsymbol{\eta} d \gamma+\int_{\Gamma} \Pi\left(\mathbf{d}_{s}\right) \mathbf{n}_{s} \cdot \boldsymbol{\eta} d \Gamma=0 \quad \forall \boldsymbol{\eta} \in U^{\lambda}
$$

\subsection{Thin-wall model}

The second major source of complexity of FSI problems results from the presence of two coupled physics: the fluid and the solid . Due to the fact that our target output are mainly driven by the haemodynamics of the system, we concentrate the computational effort on the fluid domain

In this regards, we aim at building a lower dimensional structural model, while keeping the effect that the compliant arterial wall produces on the blood flow. It is then possible to integrate the solid model as a boundary condition for the fluid one through specific assumptions on the biomechanics of the arterial wall, cf. [24, 94, 43].

We assume that the wall is a thin-wall structure, i.e. $h_{s}<<D$, being $h_{s}$ the wall thickness and $D$ the lumen diameter. Using this approximation in system (2.7), volume integrals on $\Omega_{s}$ can be substituted with surface integrals on $\Gamma$ multiplied by the thickness $h_{s}$. Furthermore, we assume that all the derivatives of structural quantities in the normal direction are negligible. In the following, the pedix $\gamma$ denotes the tangential derivatives involved in grandient operators. With respect to the notaton of 


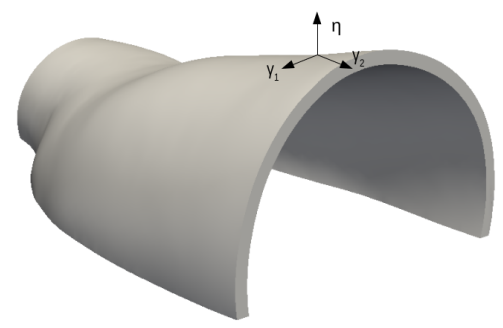

Figure 2: Schematic representation of the structural domain

Figure 2, considering a generic scalar field $\phi$, these assumptions yield the following mathematical formulas:

$$
\begin{gathered}
\phi\left(\eta, \gamma_{1}, \gamma_{2}\right) \approx \phi\left(\gamma_{1}, \gamma_{2}\right), \text { therefore } \int_{\Omega_{s}} \phi\left(\eta, \gamma_{1}, \gamma_{2}\right) d \Omega_{s}=\int_{\Gamma} h_{s} \phi\left(\gamma_{1}, \gamma_{2}\right) d \Gamma \\
\nabla_{\mathbf{x}} \phi \approx \nabla_{\gamma} \phi, \text { therefore } \nabla_{\mathbf{x}} \phi \cdot \mathbf{n} \approx 0
\end{gathered}
$$

being $\nabla_{\gamma} \phi=\nabla_{\mathbf{x}} \phi-\nabla_{\mathbf{x}} \phi \cdot \mathbf{n}$ the tangential gradient of $\phi$.

At this point, we can rewrite the structural equation of system (2.7) as follows:

$$
\int_{\Gamma} h_{s} \rho_{s} \frac{\partial^{2} \mathbf{d}_{s}}{\partial t^{2}} \cdot \mathbf{v}_{s} d \Gamma+\int_{\Gamma} h_{s} \boldsymbol{\Pi}_{\gamma}\left(\mathbf{d}_{s}\right): \nabla_{\gamma} \mathbf{v}_{s} d \Gamma=\int_{\Gamma} \boldsymbol{\Pi}_{\gamma}\left(\mathbf{d}_{s}\right) \mathbf{n}_{s} \cdot \mathbf{v}_{s} d \Gamma
$$

where $\Pi_{\gamma}\left(\mathbf{d}_{s}\right)$ is the surface stress tensor that, with respect to (2.5), is modified as follows:

$$
\boldsymbol{\Pi}_{\gamma}\left(\mathbf{d}_{s}\right)=\lambda_{s} \operatorname{tr}\left(\frac{\nabla_{\gamma} \mathbf{d}_{s}+\left(\nabla_{\gamma} \mathbf{d}_{s}\right)^{T}}{2}\right)+\mu_{s}\left(\nabla_{\gamma} \mathbf{d}_{s}+\left(\nabla_{\gamma} \mathbf{d}_{s}\right)^{T}\right) .
$$

Using the coupling conditions (3.3) and (3.4), we replace the structural unknowns in the first and third term of equation (3.5) with fluid ones:

$$
\int_{\Gamma} h_{s} \rho_{s} \frac{\partial \mathbf{u}}{\partial t} \cdot \mathbf{v}_{f} d \Gamma+\int_{\Gamma} h_{s} \boldsymbol{\Pi}_{\gamma}\left(\mathbf{d}_{s}\right): \nabla_{\gamma} \mathbf{v}_{f} d \Gamma=-\int_{\Gamma} \sigma_{f}(\mathbf{u}, p) \mathbf{n}_{f} \cdot \mathbf{v}_{f} d \Gamma .
$$

We notice that the second term of equation (3.5) still depends on structural unknowns, i.e. on the displacement $\mathbf{d}_{s}$. It is possible to recover $\mathbf{d}_{s}$ from (3.3) and approximated, e.g., by exploiting a generic discretization of the first order temporal derivatives of $\mathbf{d}_{s}$

$$
\left.\frac{\partial \mathbf{d}_{s}}{\partial t}\right|_{t=t^{n+1}} \approx \frac{\alpha}{\Delta t} \mathbf{d}_{s}^{n+1}-\mathbf{f}_{\mathbf{d}_{s}}^{n}
$$


where $\mathbf{f}_{\mathbf{d}_{s}}^{n}=f\left(\mathbf{d}_{s}^{n}, \dot{\mathbf{d}}_{s}^{n}, ..\right)$ and similarly for $\mathbf{f}_{\mathbf{u}}^{n}$. Discretization of the kinematic condition (3.3) then reads: $\mathbf{u}=\frac{\partial \mathbf{d}_{s}}{\partial t}$ on $\Gamma$ :

$$
\mathbf{u}^{n+1}=\frac{\alpha}{\Delta t} \mathbf{d}_{s}^{n+1}-\mathbf{f}_{\mathbf{d}_{s}}^{n}
$$

which then allow us to substitute $\mathbf{d}_{s}$ at the time $t^{n+1}$ inside the stress tensor $\boldsymbol{\Pi}_{\gamma}\left(\mathbf{d}_{s}^{n+1}\right)$ as follows:

$$
\boldsymbol{\Pi}_{\gamma}\left(\mathbf{d}_{s}^{n+1}\right)=\frac{\Delta t}{\alpha} \boldsymbol{\Pi}_{\gamma}\left(\mathbf{u}^{n+1}+\mathbf{f}_{\mathbf{d}_{s}}^{n}\right) .
$$

Similarly, using

$$
\left.\frac{\partial \mathbf{u}}{\partial t}\right|_{t=t^{n+1}} \approx \frac{\alpha}{\Delta t} \mathbf{u}^{n+1}-\mathbf{f}_{\mathbf{u}}^{n},
$$

the temporal discretization of equation (3.6) at time $t^{n+1}$ reads as follows

$$
\begin{array}{r}
\int_{\Gamma} h_{s} \rho_{s}\left(\frac{\alpha}{\Delta t} \mathbf{u}^{n+1}-\mathbf{f}_{\mathbf{u}}^{n}\right) \cdot \mathbf{v}_{f} d \Gamma+\int_{\Gamma} h_{s} \frac{\Delta t}{\alpha} \boldsymbol{\Pi}_{\gamma}\left(\mathbf{u}^{n+1}+\mathbf{f}_{\mathbf{d}_{s}}^{n}\right): \nabla_{\gamma} \mathbf{v}_{f} d \Gamma \\
=-\int_{\Gamma} \sigma_{f}(\mathbf{u}, p) \mathbf{n}_{f} \cdot \mathbf{v}_{f} d \Gamma
\end{array}
$$

The above equation can be seen as a generalized Robin boundary condition on $\Gamma$ for the fluid problem: in fact it states that the weak term referring to the fluid stress on $\Gamma$ can be written as a function of a boundary mass and a boundary stiffness term. We remark that $\mathbf{d}_{s}^{n}$ is not a primal unknown since it is reconstructed as a weighted sum of the velocities at previous time levels.

\subsection{Weak formulation of the reduced fluid-structure interaction problem}

We call Reduced FSI (RFSI) problem the simplification of the FSI formulation deriving from the transpiration conditions and the thin wall assumption. The RFSI system is composed by standard Navier-Stokes equation, written on a fixed domain, enhanced by a generalized Robin boundary condition on the lateral surface of the lumen. The second order differential operator on the boundary requires a higher regularity on the surface $\Gamma$ (see [70] for more details on the analysis of partial differential equations with generalized Robin boundary conditions). Thus, to garantee the well-posedeness of the weak formulation, the velocity spaces (see section 2.2) needs to be modified as follows:

$$
\widetilde{U}=\left\{\mathbf{v} \in H^{1}(\Omega)|\mathbf{v}|_{\Gamma} \in\left[H^{1}(\Gamma)\right]^{3}\right\}, \widetilde{U}_{D}=\left\{\mathbf{v} \in \widetilde{U} \mid \mathbf{v}=0 \text { on } \Gamma_{\text {fixed }}\right\} .
$$

We use P2-P1 Taylor-Hood finite element spaces, which fulfill the higher regurality requirement on the boundary $\Gamma$, i.e., $U_{h} \subset \widetilde{U}, U_{h, D} \subset \widetilde{U}_{D}$, and $Q_{h} \subset L^{2}\left(\Omega_{f}\right)$.

We suppose to divide the temporal interval $[0, T]$ in $N_{T}+1$ points uniformly distributed with a time step $\Delta t=T / N_{T}$, such that $t_{n}=n \Delta t$. The fully discretized 
weak formulation of the RFSI problem then reads: for all $n=0, . ., N_{T}-1$, find $\left(\mathbf{u}_{h}^{n+1}, p_{h}^{n+1}\right) \in U_{h} \times Q_{h}$ such that

$$
\left\{\begin{aligned}
a\left(\mathbf{u}_{h}^{n+1}, \mathbf{v}_{h}\right)+c\left(\mathbf{u}_{h}^{n}, \mathbf{u}_{h}^{n+1}, \mathbf{v}_{h}\right)+b\left(\mathbf{v}_{h}, p_{h}^{n+1}\right) & =f\left(\mathbf{v}_{h} ; \mathbf{u}_{h}^{n}, \mathbf{d}_{s, h}^{n}\right) & & \forall \mathbf{v}_{h} \in U_{h, D} \\
b\left(\mathbf{u}_{h}^{n+1}, q_{h}\right) & =0 & & \forall q_{h} \in Q_{h},
\end{aligned}\right.
$$

where

$$
\begin{aligned}
a\left(\mathbf{u}_{h}^{n+1}, \mathbf{v}_{h}\right)= & \int_{\Omega_{f}}\left(\rho_{f} \alpha \frac{\mathbf{u}_{h}^{n+1}}{\Delta t} \cdot \mathbf{v}_{h}+\mu_{f}\left(\nabla \mathbf{u}_{h}^{n+1}+\left(\nabla \mathbf{u}_{h}^{n+1}\right)^{T}\right): \nabla \mathbf{v}_{h}\right) d \Omega \\
& +\int_{\Gamma} h_{s}\left(\rho_{s} \frac{\alpha}{\Delta t} \mathbf{u}_{h}^{n+1} \cdot \mathbf{v}_{h} \frac{\Delta t}{\alpha} \mathbf{\Pi}_{\gamma}\left(\mathbf{u}_{h}^{n+1}\right): \nabla_{\gamma} \mathbf{v}_{h}\right) d \Gamma \\
c\left(\mathbf{u}_{h}^{n}, \mathbf{u}_{h}^{n+1}, \mathbf{v}_{h}\right)= & \int_{\Omega_{f}} \rho_{f}\left(\left(\mathbf{u}_{h}^{n}\right) \cdot \nabla\right) \mathbf{u}_{h}^{n+1} \cdot \mathbf{v}_{h} \\
b\left(\mathbf{v}_{h}, p_{h}^{n+1}\right)= & \int_{\Omega_{f}} p_{h}^{n+1} \nabla \cdot \mathbf{v}_{h} d \Omega \\
f\left(\mathbf{v}_{h} ; \mathbf{u}_{h}^{n}, \mathbf{d}_{s, h}^{n}\right)= & \int_{\Omega_{f}} \rho_{f} \mathbf{f}_{\mathbf{u}_{h}}^{n} \cdot \mathbf{v}_{h} d \Omega+\int_{\Gamma_{N}^{f}} \mathbf{g}_{f} \cdot \mathbf{v}_{h} d \Gamma \\
& +\int_{\Gamma} h_{s}\left(\rho_{s} \mathbf{f}_{\mathbf{u}_{h}}^{n} \cdot \mathbf{v}_{h}+\frac{\Delta t}{\alpha} \mathbf{\Pi}_{\gamma}\left(\mathbf{f}_{\mathbf{d}_{s, h}}^{n}\right): \nabla_{\gamma} \mathbf{v}_{h}\right) d \Gamma .
\end{aligned}
$$

Solutions $\left(\mathbf{u}_{h}^{n}, p_{h}^{n}\right)$ of problem (3.8) represent the so called high fidelity approximation which will be used as reference solution in the numerical reduction phase described in the next section.

\section{Reduced Order Methods for Reduced Fluid-Structure Interaction Models}

\subsection{Numerical reduction}

Once the model equations have been simplified, we are ready to proceed with their numerical reduction. In this section we will recall only some basic concepts of reduced order models, the full theoretical assessment of these methods can be found in $[61,99]$. Numerical reduction techniques are particularly interesting when dealing with parametric PDEs. The parameters can be either geometrical and/or physical, for instance the diameter of the vessel, mean flowrate, frequency, mechanical properties.

Let us suppose to select a suitable set of parameters. We denote $\boldsymbol{\mu}$ the vector that collects all the chosen coefficients and $\mathcal{D}$ the set of possible parameter realisations. The high fidelity approximations resulting from system (3.8) will thus depend not only on space and time but also on the specific values of the parameters: $\mathbf{u}_{h}^{n}(\mathbf{x} ; \boldsymbol{\mu})$ and $p_{h}^{n}(\mathbf{x} ; \boldsymbol{\mu})$ for $n=1, \ldots, N_{T}$. 
The aim of reduction techniques is to find a suitable set of basis functions to approximate the high fidelity solution by a Galerkin projection onto low dimensional spaces $U_{N}$ and $Q_{N}$. We therefore focus on the construction of discretization spaces $U_{N} \subset U_{h}^{g}, U_{N, D} \subset U_{h, D}^{g}$ and $Q_{N} \subset Q_{h}$, whose dimensions are orders of magnitude lower than the dimension of the finite element spaces. The dimension of the velocity and pressure spaces $U_{N} \times Q_{N}$ is noted by $N$ and the one of $U_{h}^{g} \times Q_{h}$ by $\mathcal{N}_{h}$. The weak formulation of the RFSI problem in the reduced numerical framework reads: for all $n=0, \ldots, N_{T}-1$, find $\left(\mathbf{u}_{N}^{n+1}, p_{N}^{n+1}\right) \in U_{N} \times Q_{N}$ such that

$$
\left\{\begin{aligned}
a\left(\mathbf{u}_{N}^{n+1}, \mathbf{v}_{N}\right)+c\left(\mathbf{u}_{N}^{n}, \mathbf{u}_{N}^{n+1}, \mathbf{v}_{N}\right)+b\left(\mathbf{v}_{N}, p_{N}^{n+1}\right) & =f\left(\mathbf{v}_{N} ; \mathbf{u}_{N}^{n}, \mathbf{d}_{s, N}^{n}\right) & & \forall \mathbf{v}_{N} \in U_{N, D} \\
b\left(\mathbf{u}_{N}^{n+1}, q_{N}\right) & =0 & & \forall q_{N} \in Q_{N},
\end{aligned}\right.
$$

We remark that to build a basis for the spaces $U_{N}$ and $Q_{N}$, we need to explore the variability of the finite element solutions with respect to both the time and the parameters.

\subsection{Proper Orthogonal Decomposition}

To generate a set of basis functions for the spaces $U_{N}$ and $Q_{N}$ we rely on the Proper Orthogonal Decomposition (POD) based on snapshots temporal solutions. In the context of this work we only detail the specific numerical choices that we perform to reduce the RFSI problem, for more details about POD algorithm applied to fluid problems the interested reader can find more details in, e.g., [104, 72, 126]. The POD is performed assuming a fix value for each parameter in $\boldsymbol{\mu}$, which will be thus removed from the notation.

Let us suppose that our solution is periodic and that the time indices $n=0, \ldots, N_{T}$ are related to temporal instants spanning one single period. We select as snapshots the high fidelity solutions of problem (3.8) sampled at specific instants such that we cover the time lapse of one full cycle. To reduce the size of the eigenvalue problem involved in the POD reduction, the solutions are sampled at a lower frequency than the time discretization used in the simulation. Instead of using all of the $N_{T}+1$ solutions, only a fraction $N_{S}=N_{T} / k$ are taken. The set of selected temporal indeces is therefore $\mathcal{N}_{S}=\left\{0, k, 2 k, 3 k, \ldots, N_{T}\right\}$. The solutions $\left\{\left(\mathbf{u}_{h}^{s}, p_{h}^{s}\right)\right\}_{s \in \mathcal{N}_{S}}$ will represent our snapshots, the starting point for the POD analysis. Since velocity and pressure have different dynamics, we split the POD into two separate eigenvalue decompositions as also done in [51]. We measure the energy associated to the snapshots using the following scalar products: for the velocity, we use

$$
\left(\mathbf{u}_{h}, \mathbf{v}_{h}\right)_{U_{h}^{g}}:=\int_{\Omega^{f}} \nabla \mathbf{u}_{h}: \nabla \mathbf{v}_{h} d \Omega+\int_{\Gamma} \nabla_{\gamma} \mathbf{u}_{h}: \nabla_{\gamma} \mathbf{v}_{h} d \Gamma
$$

and for the pressure,

$$
\left(p_{h}, q_{h}\right)_{Q}:=\int_{\Omega} p_{h} q_{h} d \Omega
$$


Then, we compute the two Gramian matrices

$$
G_{i j}^{\mathbf{u}}=\left(\mathbf{u}_{h}^{i}, \mathbf{u}_{h}^{j}\right)_{\mathbf{v}} \quad \text { and } \quad G_{i j}^{p}=\left(p_{h}^{i}, p_{h}^{j}\right)_{Q} \quad \forall j, i \in \mathcal{N}_{S}
$$

We remark that since we deal with RFSI problems, the internal product of the velocity space is enhanced by a boundary term. At this stage the indices $i, j$ are not contiguous. Since $\mathcal{N}_{S}$ is ordered, it is easy to renumber the indices such that $G^{\mathbf{u}}$ and $G^{p}$ are $N_{S} \times N_{S}$ matrices. We perform the eigenvalue decomposition of $G^{\mathbf{u}}$ and the one of $G^{p}$, obtaining the pairs $\left(\lambda_{k}^{\mathbf{u}}, \boldsymbol{\zeta}_{k}^{\mathbf{u}}\right)$ and $\left(\lambda_{k}^{p}, \boldsymbol{\zeta}_{k}^{p}\right)$ where $\lambda_{k}^{\mathbf{u}}, \lambda_{k}^{p} \in \mathbb{R}$ and $\boldsymbol{\zeta}_{k}^{\mathbf{u}}, \boldsymbol{\zeta}_{k}^{p} \in \mathbb{R}^{N_{S}}$ are the $k-t h$ eigenvalues and eigenvectors of the velocity and pressure Gramian matrices, respectively, for $k=1, \ldots, N_{S}$; we sort the eigenvalues from the largest to the smallest. Fixing the same tolerance $0<\delta<1$ for both velocity and pressure decompositions, we select the first $N^{\mathbf{u}}$ and $N^{p}$ eigenpairs such that:

$$
\frac{\sum_{j=1}^{N^{\mathbf{u}}} \lambda_{j}^{\mathbf{u}}}{\sum_{k=1}^{N_{S}} \lambda_{k}^{\mathbf{u}}} \geq 1-\delta \quad \text { and } \quad \frac{\sum_{j=1}^{N^{p}} \lambda_{j}^{p}}{\sum_{k=1}^{N_{S}} \lambda_{k}^{p}} \geq 1-\delta,
$$

respectively. The $j$-th velocity eigenfunction $\phi_{j}^{\mathbf{u}} \in U_{h}^{g}$ is reconstructed using the linear combination:

$$
\phi_{j}^{\mathbf{u}}=\frac{1}{\lambda_{j}} \sum_{s \in \mathcal{N}_{S}}\left[\boldsymbol{\zeta}_{j}^{\mathbf{u}}\right]_{s} \mathbf{u}_{h}^{s}, \quad \text { for } j=1, \ldots, N^{\mathbf{u}}
$$

and similarly for $\phi_{j}^{p} \in Q_{h}, j=1, \ldots, N^{p}$. The velocity bases are linear combinations of solutions of problem (3.8), thus, they all verify: $\int_{\Omega} q_{h} \nabla \cdot \phi_{j}^{\mathbf{u}}=0, \forall q_{h} \in Q_{h}$ for $j=1, \ldots, N^{\mathbf{u}}$. If we consider the functional spaces generated from the velocity functions $\phi_{j}^{\mathbf{u}}$ and the pressure modes $\phi_{j}^{p}$, linear system (4.1) would be singular. For Navier-Stokes systems, this issue can be solved by restricting the system and solving it only for the velocity unknown (see e.g., [20]). Unfortunately, this is not possible when considering problem (3.8). Indeed, the boundary condition applied on $\Gamma$ derives from a structural model whose solution is driven by the pressure condition set on the external boundary of the structural model itself (see [24, 90]). If in the reduced system (4.1) we do not account for the pressure variable, we cannot recover the correct velocity on the boundary $\Gamma$ and the related output functionals (e.g., wall shear stresses). In order to construct a non singular reduced system (4.1), for each selected pressure mode $\phi_{j}^{p}$, we define the corresponding supremizer function $\boldsymbol{\sigma}_{j} \in U_{h, D}^{g}$ as the solution of the following problem (see [107]):

$$
\left(\boldsymbol{\sigma}_{j}, \mathbf{v}_{h}\right)=\int_{\Omega} \phi_{j}^{p} \nabla \cdot \mathbf{v}_{h} d \Omega \quad \forall \mathbf{v}_{h} \in U_{h}^{g}, \quad \text { for } j=1, \ldots, N^{p},
$$

and we add the supremizers to the POD velocity basis. We define $U_{N}$ and $Q_{N}$ the POD reduced spaces associated to the RFSI model that are generated by the basis functions $\left\{\boldsymbol{\phi}_{j}^{\mathbf{u}}\right\}_{j=1}^{N^{\mathbf{u}}} \oplus\left\{\boldsymbol{\phi}_{j}^{\boldsymbol{\sigma}}\right\}_{j=1}^{N^{p}}$ and $\left\{\phi_{j}^{p}\right\}_{j=1}^{N^{p}}$, respectively, where $\boldsymbol{\phi}_{j}^{\boldsymbol{\sigma}}$ for $j=1, \ldots, N^{p}$ represent 
the orthonormalization of the supremizer functions $\boldsymbol{\sigma}_{j}$, obtained with a Gram-Schmidt algorithm with respect to the scalar product $(\cdot, \cdot)_{U_{h}^{g}}$. The other basis functions are already orthogonal thanks to the POD algorithm. The total dimension of the reduced system is $N=N^{\mathbf{u}}+2 \cdot N^{p}$.

\section{Fluid structure interaction in a patient-specific aortofemoral artery}

In this section we address the study of the blood flow dynamics in a patient-specific aortofemoral artery. Studying the hemodynamics of the vasculature distal to the abdominal aorta may be important in understanding common diseases in peripheral arteries downstream of the thoracic aorta. Diseases in the peripheral vasculature affect millions of people in the U.S and can have a profound effect on daily quality of life. Peripheral arterial disease is the build-up of fatty tissue, or atherosclerosis, in lower extremity arteries. The prevalence of peripheral arterial occlusive disease increases with age and can increase to up to $20 \%$ of the geriatric population. Atherosclerotic occlusive disease of the lower extremity arteries is a major cause of walking impairment, pain, ulcerations and gangrene.

The geometry of the aortofemoral artery used for the numerical simulations has been obtained from [1] and is shown in Figure 3, while the meshes for the fluid and structure physical domains were generated using a software library [39] based on VMTK. We consider the blood characterized by a density $\rho_{f}=1.06 \mathrm{~g} / \mathrm{cm}^{3}$ and by a dynamic viscosity $\mu_{f}=0.04 \mathrm{~g} /(\mathrm{cm} \mathrm{s})$. The physical parameters for the structure are set as follows: Young's modulus $E_{s}=4 \cdot 10^{6} \mathrm{dyne} / \mathrm{cm}^{2}$, Poisson's ratio $\nu_{s}=0.45$ and density $\widehat{\rho}_{s}=1.2 \mathrm{~g} / \mathrm{cm}^{3}$. The boundaries of the computational fluid domain are reported in Figure $3 b, 3 c$ and $3 d$ (see Table 1 for the identification of each artery). The inflow section of the fluid domain correspond to surface ID 1 in Figure $3 b$ and represents the aortic root.

In a pre-processing phase, starting from the geometry shown in Figure 3 we generated flow extensions at the inflow 1 to obtain an inflow section of circular shape, see Figure 4, where a patient-specific flowrate is prescribed using a Dirichlet boundary condition, i.e., mapping the flowrate through a parabolic velocity profile. The flowrate profile prescribed is shown in Figure 5. At each fluid outflow we impose the following $R C$-type boundary condition proposed in [55]:

$$
\boldsymbol{\sigma}^{n+1} \mathbf{n}_{f}=\frac{\Delta t}{\Delta t+R C}\left(R Q^{n}+P^{n} \frac{R C}{\Delta t}\right),
$$

where $\Delta t=10^{-3} \mathrm{~s}$ is time step size used in our simulations, $P^{n}$ and $Q^{n}$ are the pressure and flowrate values, respectively, at time $t^{n}=n \Delta t$. In equation (5.1) $R$ and $C$ represent the resistance and capacity associated to a distal position of the vascular tree. In Table 1 we report the numerical values of the resistances used; for each outflow, the value of the capacity used is $C=3 \Delta t \backslash R$. 


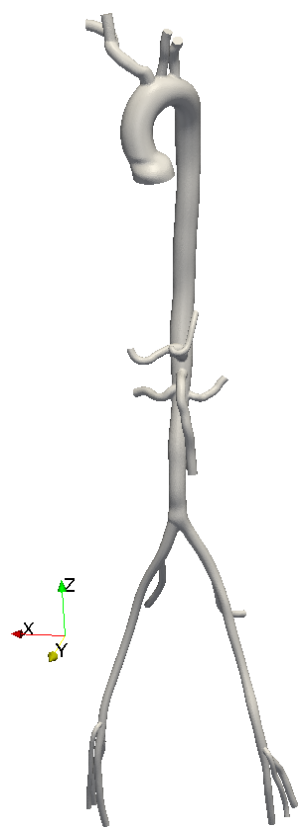

(a) Whole geometry.

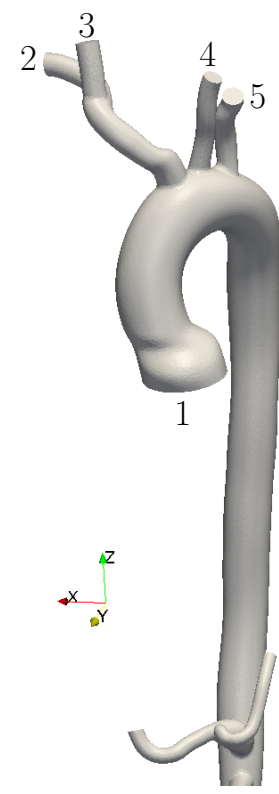

(b) Thoracic aorta.

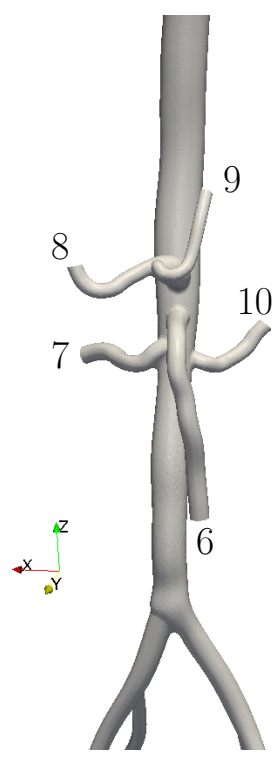

(c) Abdominal aorta.

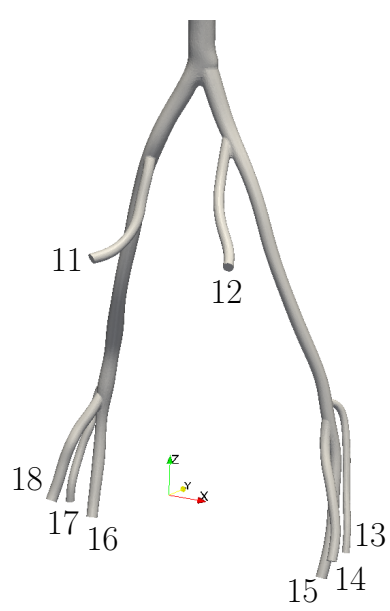

(d) Iliac and femoral arteries.

Figure 3: Patient-specific aortofemoral geometry: from left to right we show the whole arterial geometry and a zoomed-in view of the thoracic and descending aorta, of the abdominal aorta and of the iliac arteries.

The solid domain is clamped at the inlet and outflow rings while at the external wall we impose the following Robin-like boundary condition [90, 83] of the type:

$$
p_{0} \widehat{\mathbf{n}}_{s}+\widehat{\Pi} \widehat{\mathbf{n}}_{s}+\kappa_{s} \widehat{\mathbf{d}}_{s}+c_{s} \frac{\partial \widehat{\mathbf{d}}_{s}}{\partial t}=0 \quad \text { on } \widehat{\Gamma}_{e x t}^{s}
$$

where $\kappa_{s} \widehat{\mathbf{d}}_{s}$ and $c_{s} \frac{\partial \widehat{\mathbf{d}}_{s}}{\partial t}$ represent the elastic and the viscoelastic components of the external tissue, respectively. Concerning the reduced structural model, equation (3.7) is modified as follows:

$$
\begin{aligned}
\int_{\Gamma}\left(\frac{h_{s} \rho_{s} \alpha}{\Delta t}+\right. & \left.\kappa_{s} \frac{\Delta t}{\alpha}+c_{s}\right) \mathbf{u}^{n+1} \cdot \mathbf{v}_{f} d \Gamma+\frac{\Delta t}{\alpha} \int_{\Gamma} h_{s} \mathbf{\Pi}_{\gamma}\left(\mathbf{u}^{n+1}+\mathbf{f}_{\mathbf{d}}^{n}\right): \nabla_{\gamma} \mathbf{v}_{f} d \Gamma \\
& =\int_{\Gamma}\left(-\sigma_{f}\left(\mathbf{u}^{n+1}, p^{n+1}\right) \mathbf{n}+h_{s} \rho_{s} \mathbf{f}_{\mathbf{u}}^{n}\right) \cdot \mathbf{v}_{f} d \Gamma-\int_{\Gamma} \kappa_{s} \frac{\Delta t}{\alpha} \mathbf{f}_{\mathbf{d}}^{n} \cdot \mathbf{v}_{f} d \Gamma
\end{aligned}
$$




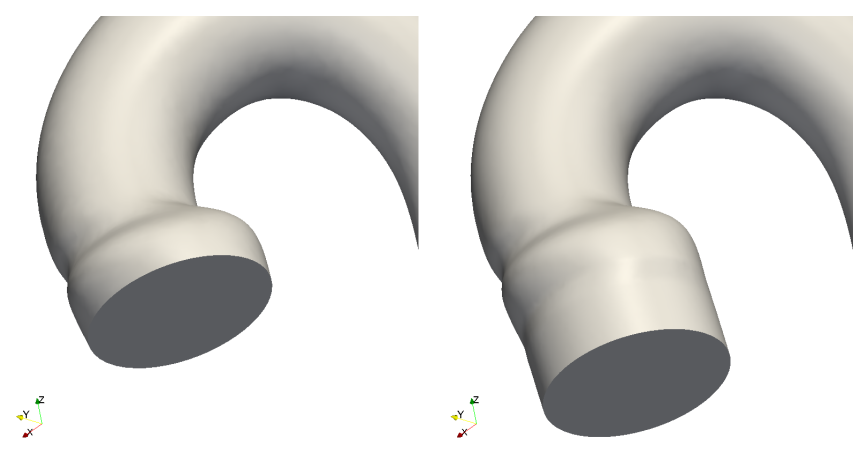

Figure 4: Initial geometry (left) and flow extension generated at the inflow section of the fluid domain (right).

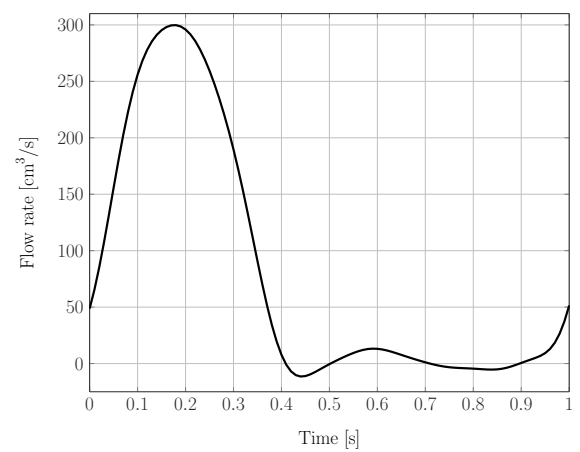

Figure 5: Patient-specific inflow flowrate profile prescribed.

\subsection{Numerical results obtained by the full three dimensional FSI model}

In this section we show the results obtained using the full 3D FSI model described in section 2. In the three-dimensional setting, we set the thickness of the arterial wall 15 $\%$ of to the local radius of the lumen.

The details of the computational meshes realized for the fluid and solid domains are summarized in Table 2 while the number of degrees of freedom obtained after spatial discretization is reported in Table 3. The numerical simulations have been carried out using PizDora, a Cray XC 40 supercomputer at the Swiss National Supercomputing center, whose technical specifications are summarized in Table 4.

In Figure 6 and 7 we show a post-processing of the solution obtained at several time instances during the third heart beat simulated. In particular in Figure 6 we show a volume rendering of the blood flow velocity while in Figure 7 we report the displacement of the the arterial wall.

The blood flow features transition to turbulent flow developing in the downstream part of the descending aorta. The typical peak Reynolds number $\operatorname{Re} \approx 2^{\prime} 650$, based 


\begin{tabular}{c|c|c} 
ID surface & Artery name & Resistance $\left[\right.$ dyne $\mathrm{s} \mathrm{cm}^{-5}$ ] \\
\hline 2 & Right Subclavian & 788.82 \\
\hline 3 & Right Common Carotid & $6^{\prime} 697.69$ \\
\hline 4 & Left Common Carotid & $6^{\prime} 708.98$ \\
\hline 5 & Left Subclavian & 788.87 \\
\hline 6 & Superior Mesenteric & 829.30 \\
\hline 7 & Right Renal & $4^{\prime} 141.67$ \\
\hline 8 & Hepatic & $1^{\prime} 114.61$ \\
\hline 9 & Splenic & $1^{\prime} 086.33$ \\
\hline 10 & Left Renal & $4^{\prime} 102.96$ \\
\hline 11 & Left Internal & $3^{\prime} 108.73$ \\
\hline 12 & Right Internal & $3^{\prime} 109.10$ \\
\hline 13 & Left Profunda & $6^{\prime} 620.49$ \\
\hline 14 & Right External Circumflex & $3^{\prime} 299.25$ \\
\hline 15 & Right Femoral & $3^{\prime} 312.96$ \\
\hline 16 & Left Femoral & $3^{\prime} 322.05$ \\
\hline 17 & Left Profunda & $6^{\prime} 620.49$ \\
\hline 18 & Left External Circumflex & $3^{\prime} 316.24$ \\
\hline
\end{tabular}

Table 1: Values of the resistances used at each outflow sections of the fluid domain, values obtained from [1].

on an average velocity $U=100 \mathrm{~cm} / \mathrm{s}$ and length $D=1 \mathrm{~cm}$. The very fine mesh and the SUPG-VMS model allow to recover peculiarities of the transitional flow regime. We notice the presence of several ricirculation zones, as shown in Figure 8, occurring between the end of the systolic phase and the diastolic one. To better visualize the complex flow pattern of the blood flow as well as its transitional regime, we report in Figure 9 the coherent vortex structures of the fluid flow identified by means of the Q criterion at different time frames during the third heart beat simulated.

The simulations have been done with the finite element library LifeV [16]. The strong scalability results of the parallel solver are reported in Figure 10. In the left plot of Figure 10 we report the average number of GMRES iterations per linear solve over the simulation, while in the right plot the average time to compute a single time step. It includes: computation of the nonlinear residual, assembly of the Jacobian matrix, construction of the preconditioner and numerical solution of the Jacobian linear system. We remark that in our simulations, since the harmonic extension and the structure matrices are constant, the preconditioners $\mathcal{H}_{\mathcal{S}}$ and $\mathcal{H}_{\mathcal{G}}$ defined in Section 2.5 


\begin{tabular}{cc|cc}
\multicolumn{2}{c|}{ Fluid mesh } & \multicolumn{2}{c}{ Structure mesh } \\
\hline \# Vertices & \# Tetrahedra & \# Vertices & \# Tetrahedra \\
\hline 4'237'118 & $255^{\prime} 925$ '318 & 1'525'185 & 7'311'192
\end{tabular}

Table 2: Details of the meshes used for the numerical simulations.

\begin{tabular}{ccccc} 
Fluid DoF & Structure DoF & Coupling DoF & Geometry DoF & Total \\
\hline $16^{\prime} 948^{\prime} 472$ & $4^{\prime} 575^{\prime} 555$ & $912^{\prime} 591$ & 12’711'354 $^{\prime}$ & 35'147'972
\end{tabular}

Table 3: Number of Degrees of Freedom (DoF) obtained after spatial discretization.

\begin{tabular}{ll}
\hline Number of compute nodes & 1,256 \\
Processor & 64-bit Intel Xeon processor \\
& E5-2690v3-Haswell \\
Memory & 64GB per node in 1,192 nodes \\
& 128GB per node in 64 nodes \\
Memory bandwidth & Up to 137 GB/s per node \\
Network & Dragonfly interconnect \\
\hline
\end{tabular}

Table 4: PizDora Cray XC40 technical data.

are built only once and stored at the beginning. As shown in Figure 10, we observe the robustness of the solver with respect to the number of cores utilized. Indeed, for the problem at hand, the number of linear solver iterations ranges from roughly 75 (with 512 cores) up to 85 iterations (with 4'096 cores). The average time to compute a single time step is almost perfect scalabile up to 2'048 cores; with this number of cores, a complete heartbeat therefore takes about 10 hours 


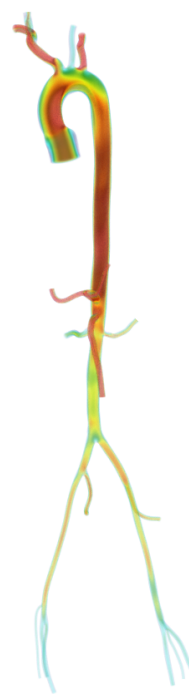

(a) $t=2.15 \mathrm{~s}$.

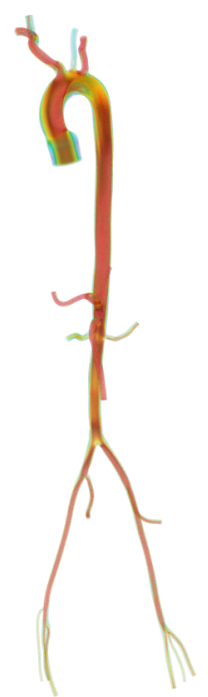

(b) $t=2.2 \mathrm{~s}$.

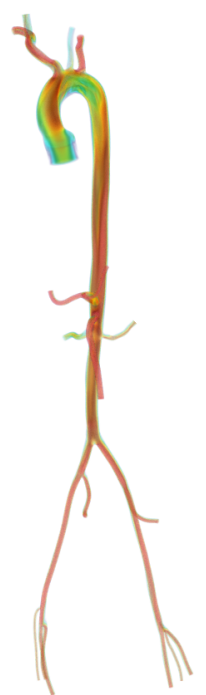

(c) $t=2.4 \mathrm{~s}$.

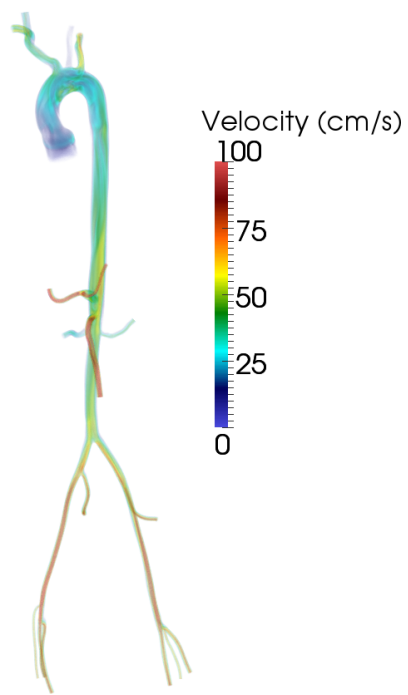

(d) $t=2.6 \mathrm{~s}$.

Figure 6: Volume rendering of the magnitude of the fluid velocity at several time instances during the third heart beat simulated.

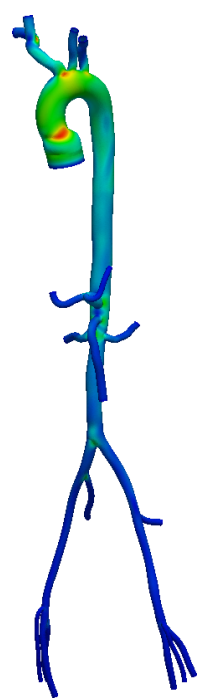

(a) $t=2.15 \mathrm{~s}$.

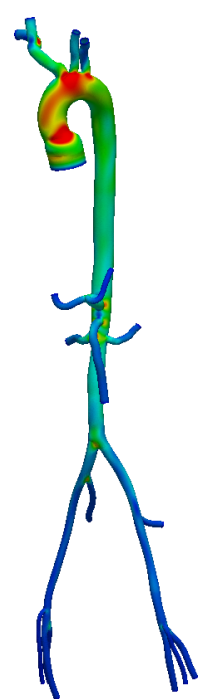

(b) $t=2.2 \mathrm{~s}$.

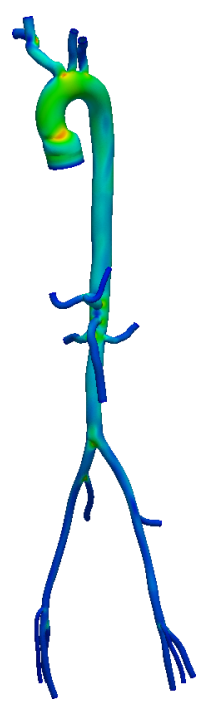

(c) $t=2.4 \mathrm{~s}$.

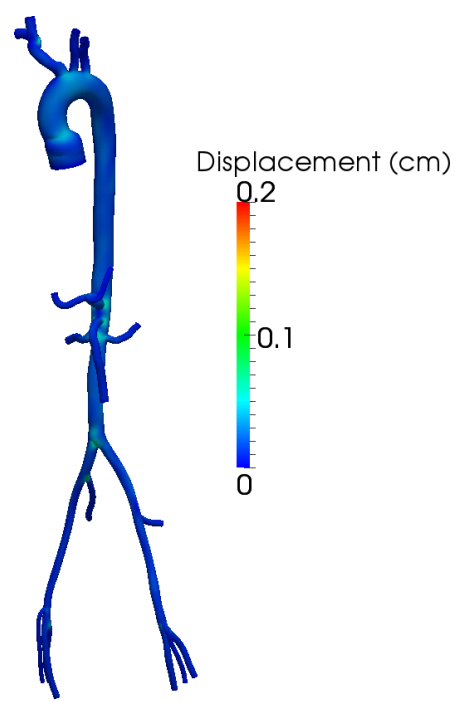

(d) $t=2.6 \mathrm{~s}$.

Figure 7: Solid displacement at several time instances during the third heart beat simulated. 


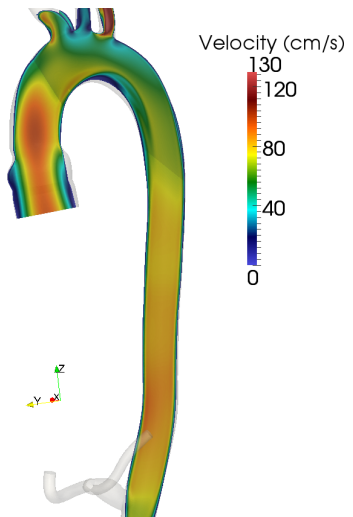

(a) Systolic peak.

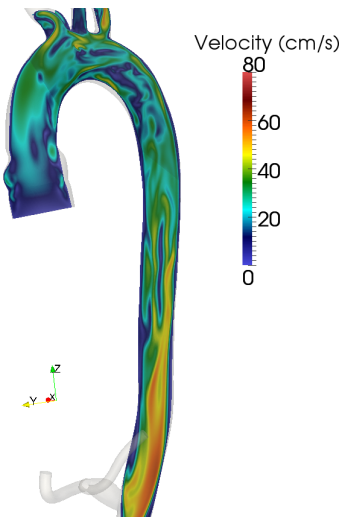

(b) End systole.

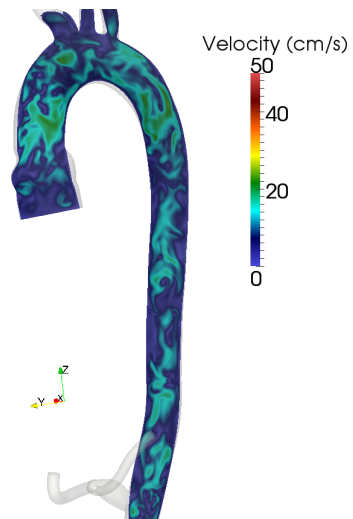

(c) Diastole.

Figure 8: Magnitude of the fluid velocity in the descending aorta at the systolic peak, at the end of systole and at diastole.

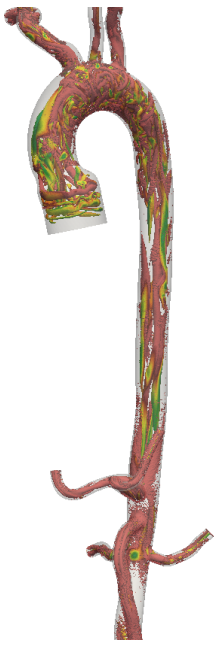

(a) $t=2.2 \mathrm{~s}$.

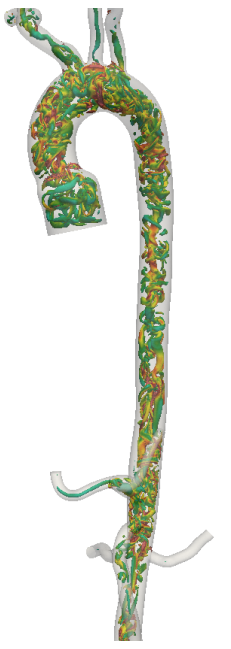

(b) $t=2.4 \mathrm{~s}$.

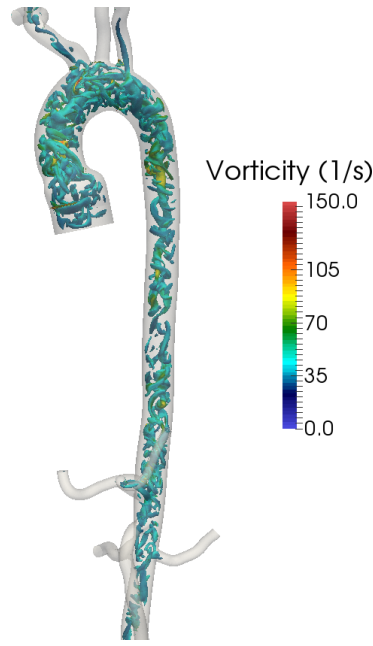

(c) $t=2.6 \mathrm{~s}$.

Figure 9: Coherent vortex structures identified by means of the $\mathrm{Q}$ criterion in the descending aorta. 

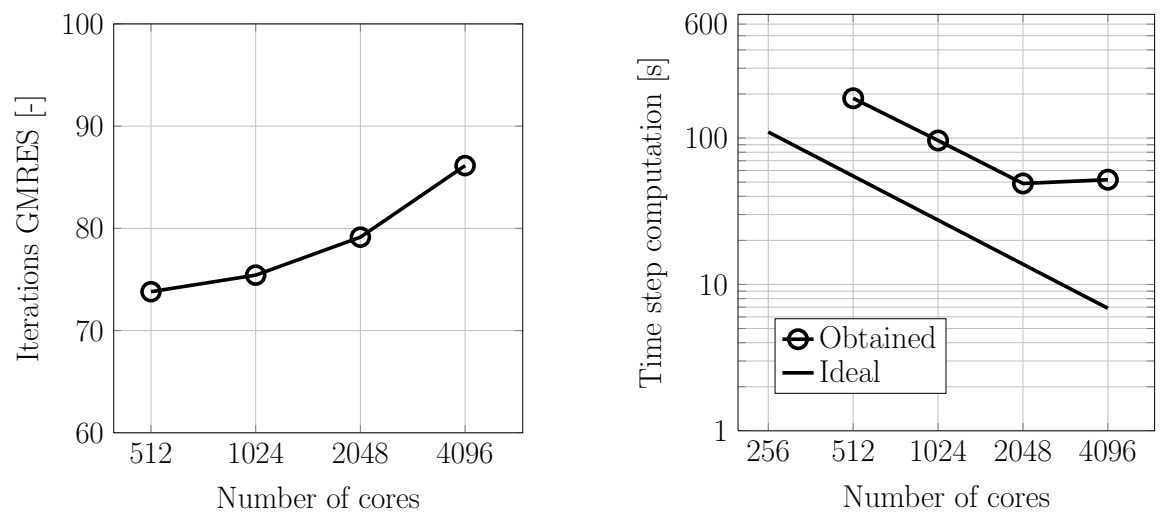

Figure 10: Scalability results: average number of GMRES iterations (left) and average time to compute a single time step. 


\subsection{Numerical simulation setting for the RFSI}

With the aim of performing a numerical reduction of the RFSI problem, we require to take ad hoc choices concerning the high fidelity discretization. During the offline phase of the reduction algorithm several finite element vectors and matrices need to be stored and manipulated, we therefore prefer to lower the dimension of the finite element spaces with respect to the ones used for three dimensional FSI solver. We use a coarser grid for the discretization of the fluid domain with a thin boundary layer of elements near the interface $\Gamma$ to maintain a good resolution towards the interface. We neglect the VMS modeling and use the stable P2-P1 finite elements pair for the discretization of the fluid system, such that no further stabilization terms are required in the formulation of the RFSI problem. This choices results in an approximation space with 8'903'292 degrees of freedom for the velocity field and 379' 160 degrees of freedom for the pressure field. Also the discretization of the temporal derivatives differs between the FSI and the RFSI solver, which moreover do not take into account turbulence models. Note however that we choose to use an implicit Euler time integrator which is dissipative, with a time step equal to $0.001 \mathrm{~s}$. Finally, regarding the physical modelling, we use a homogeneous thickness $h_{s}=0.1$ and homogeoneous coefficients $c_{s}=\kappa_{s}=10^{5}$ for the surrounding tissue since they are both important for the stability estimates of the RFSI model [23].

We choose as model parameter the structural Young modulus E. The numerical reduction is prepared with two different values of the parameters and two simulations with the finite element method for some heartbeats. We then apply twice the POD considering the two different sets of snapshots.

The set of snapshots of the first POD is computed fixing $E_{1}:=10^{6} \mathrm{dyn} / \mathrm{cm}^{2}$. The high fidelity RFSI simulation is performed for three heartbeats in order to reach a periodic state. The snapshots are then selected among the finite element solutions of the third heartbeat sampling the one every 5 time levels. The corresponding eigenvalue problem has dimension 200. Fixing a tolerance equal to $\delta=0.001$, which selects the first 48 velocity modes and the first 2 pressure ones. This remarkable difference in the modes number is due to the different behavior of the eigenvalue decays as it is shown in Figure 11. Adding the supremizers corresponding to the pressure modes, the reduced space resulting from the first POD produces 52 basis functions.

The second POD is performed considering a Young modulus equal to $E_{2}:=4 \cdot 10^{6}$ $\mathrm{dyn} / \mathrm{cm}^{2}$. As before, we focus on the high fidelity solutions of the third heartbeat that, this time, are sampled one every 10 time levels. This choice results in an eigenvalue problem of dimension equal to 100 . With a tolerance $\delta=0.01$, the POD selects 15 velocity eigenmodes and 2 pressure ones. We construct the corresponding 19 basis functions, which are orthonormalized with respect to the basis obtained with the first POD using a Gram-Schimdt algorithm.

The final reduced space has dimension 71 which is five orders of magnitude smaller than the dimension of the high fidelity approximation space. 
We suppose to use as a-posteriori error indicator the dual norm of the following residuals:

$$
\begin{aligned}
r_{N}^{n+1}\left(\left(\mathbf{v}_{h}, q_{h}\right) ; \boldsymbol{\mu}\right):= & f\left(\mathbf{v}_{h} ; \mathbf{u}_{N}^{n}, \mathbf{d}_{s, N}^{n}\right)-a\left(\mathbf{u}_{N}^{n+1}, \mathbf{v}_{h}\right) \\
& -c\left(\mathbf{u}_{N}^{n}, \mathbf{u}_{N}^{n+1}, \mathbf{v}_{h}\right)-b\left(\mathbf{v}_{h}, p_{N}^{n+1}\right)-b\left(\mathbf{u}_{N}^{n+1}, q_{h}\right),
\end{aligned}
$$

being $n=0, \ldots, N_{T}$. In Figure 12(a) we show the dual norms rescaled by the $L^{\infty}\left([0, T], \widetilde{U}\left(\Omega_{f}\right)\right)$ norm of the solution, for all the time levels of the third heartbeat, for both the values of $E_{1}$ and $E_{2}$ used to constuct the reduced order model. Even if the number of basis functions associated with $E_{2}$ is smaller than the one related to $E_{1}$, the values of the dual norms are comparable for both the choices of the Young modulus.

We select 500 random values of $\boldsymbol{\mu}$ in the interval $\left[10^{6}, 4 \cdot 10^{6}\right]$ and for each of them we compute the maximum of the dual norm of the residuals among the time levels $n=0, \ldots, N_{T}$. The values of the maximum residual dual norms shown in Figure 12-(b) are of the same orders of the ones computed for $E_{1}$ and $E_{2}$. Our reduced order model can be thus used to simulate the entire interval of parameter values $E \in\left[10^{6}, 4 \cdot 10^{6}\right]$.

We focus now on the results obtained with $E=4 \cdot 10^{6} \mathrm{dyn} / \mathrm{cm}^{2}$ and we compare the high fidelity solutions and the reduced order ones at different time levels. In Figures 13 and 14 we notice the good agreement between the two discretization for both the velocity and the pressure fields, especially during the systolic phase. In the diastolic phase the differences between the two discretization are higher since the energy associated to these time levels is smaller. In Figure 15 we compare the evolution of the averaged magnitude of wall shear stresses in three different areas. Once again, we notice that the results obtained with the high fidelity approximation and the reduced order one are in good agreement.

Concerning the computational costs, the solution of one single time instant of the RFSI solver takes $40 \mathrm{~s}$ on 720 processors, i.e., 11 hours for one heartbeat. The offline computation of the reduced order model with 71 basis functions takes about 1 hour on 720 processors, plus, roughly, 7 hours to compute the reduced structures for the online evaluation of the dual norm of the residuals. The solution of the RFSI problem for 3 heartbeats using the reduced space of dimension 71 takes 3.4 s on one single processor of a laptop. 


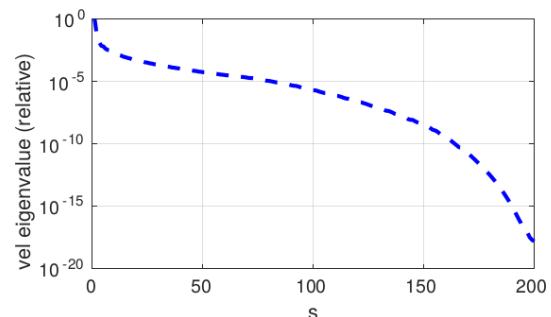

(a) velocity

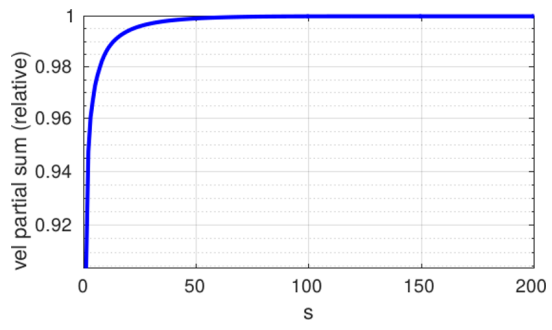

(c) velocity

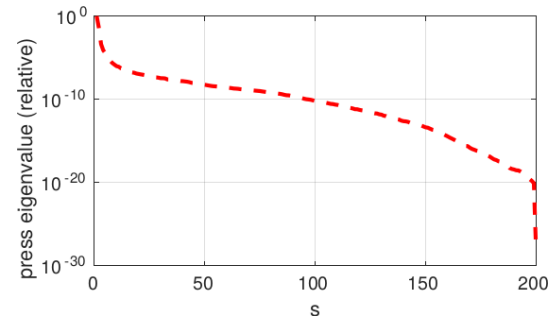

(b) pressure

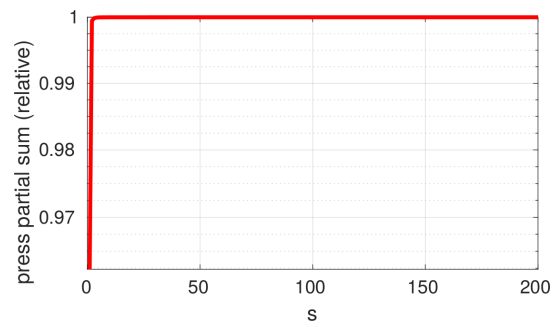

(d) pressure

Figure 11: Top: decay of POD eigenvalues computed with 200 snapshots with $E=10^{6} \mathrm{dyn} / \mathrm{cm}^{2}$. Bottom: partial sum of the POD eigenvalues computed with 200 snapshots with $E=10^{6} \mathrm{dyn} / \mathrm{cm}^{2}$.

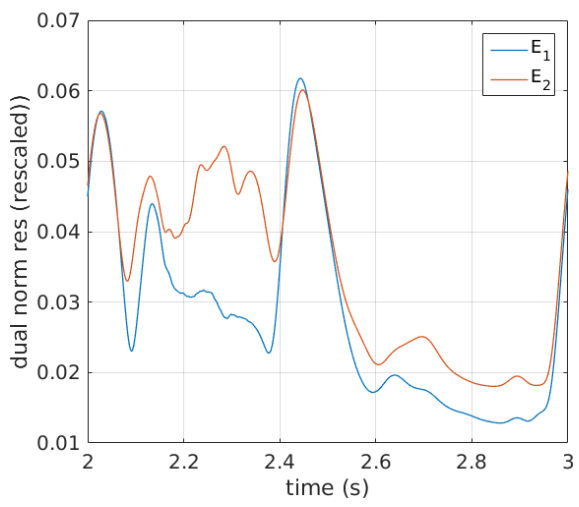

(a) $E_{1}, E_{2}$

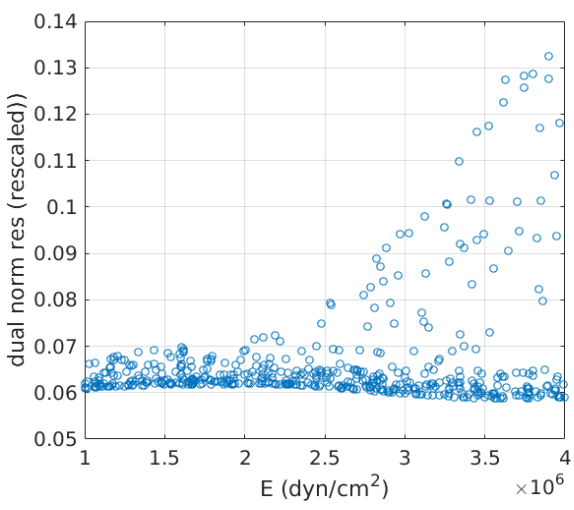

(b)

Figure 12: (a): comparisons between dual norm of the residuals obtained solving the ROM-RSFI with $E_{1}$ and $E_{2}$. (b): maximum of the dual norm of the residuals among the time levels of the third heartbeat computed for several values of the Young Modulus $E \in\left[10^{6}, 4 \cdot 10^{6}\right] \mathrm{dyn} / \mathrm{cm}^{2}$. 


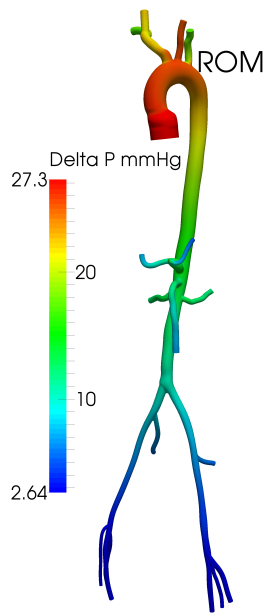

(a) time : $2.05 \mathrm{~s}$

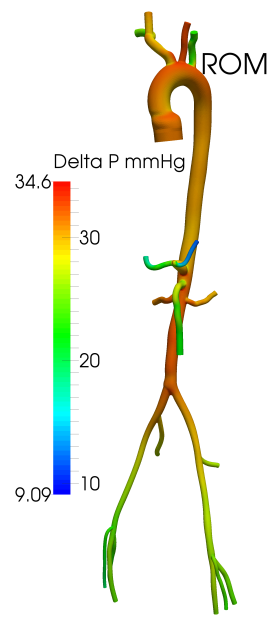

(c) time : $2.25 \mathrm{~s}$
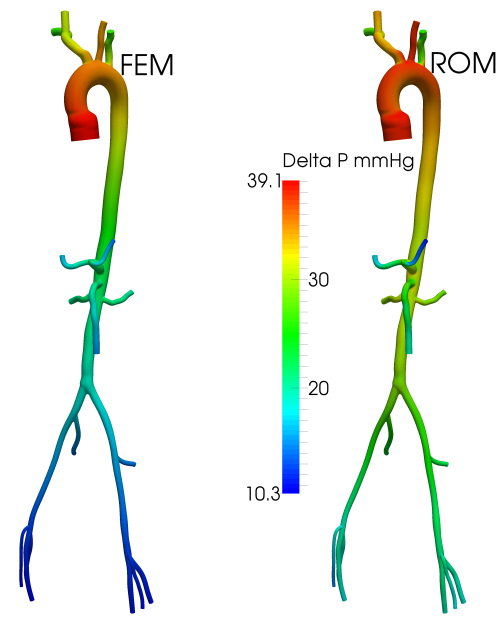

(b) time : $2.15 \mathrm{~s}$
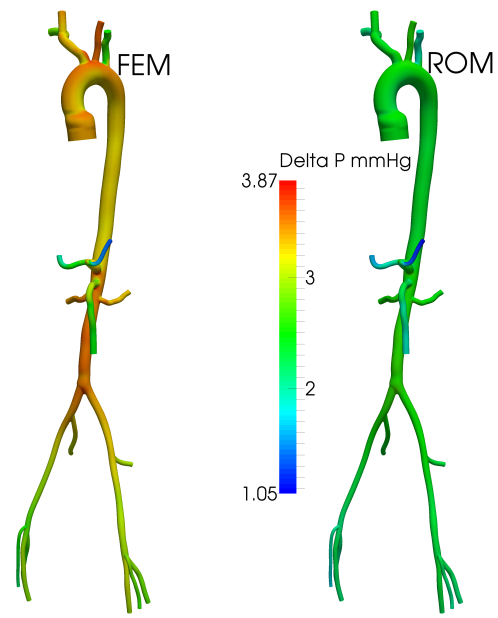

(d) time $: 2.5 \mathrm{~s}$
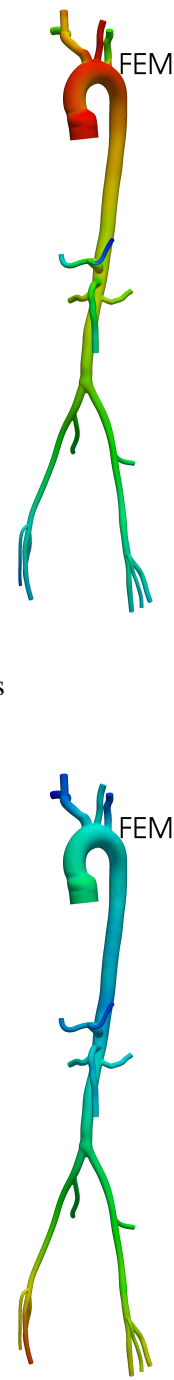

Figure 13: Comparisons between pressure solutions computed for $E=4 \cdot 10^{6} \mathrm{dyn} / \mathrm{cm}^{2}$ using the reduced order model with 71 basis functions (left) and the finite element one (right). 


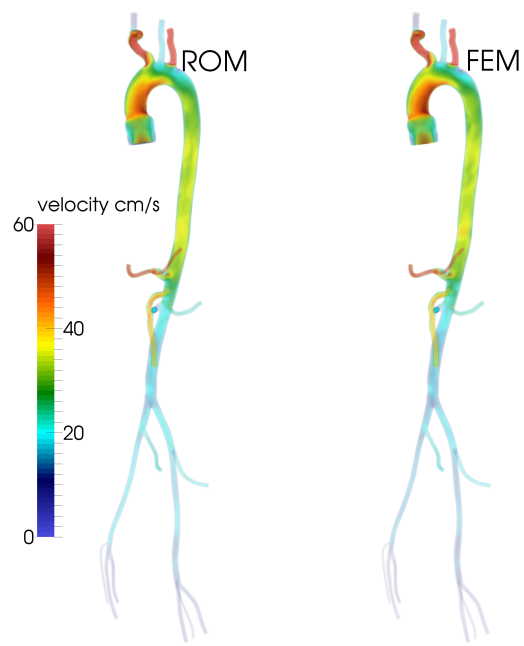

(a) time : $2.05 \mathrm{~s}$

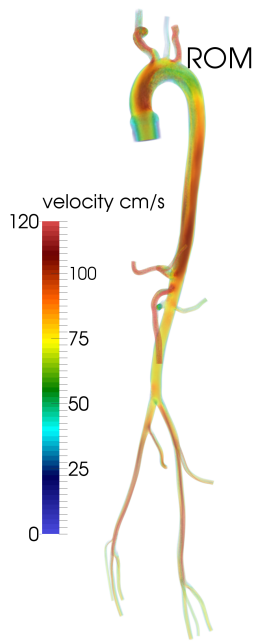

(c) time : $2.25 \mathrm{~s}$

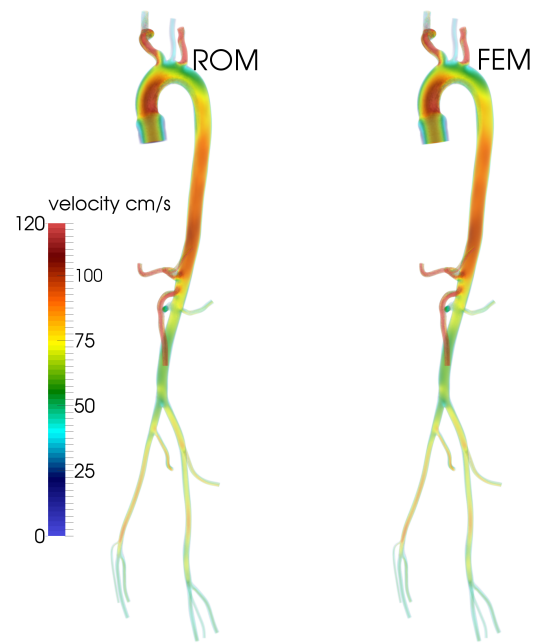

(b) time : $2.15 \mathrm{~s}$

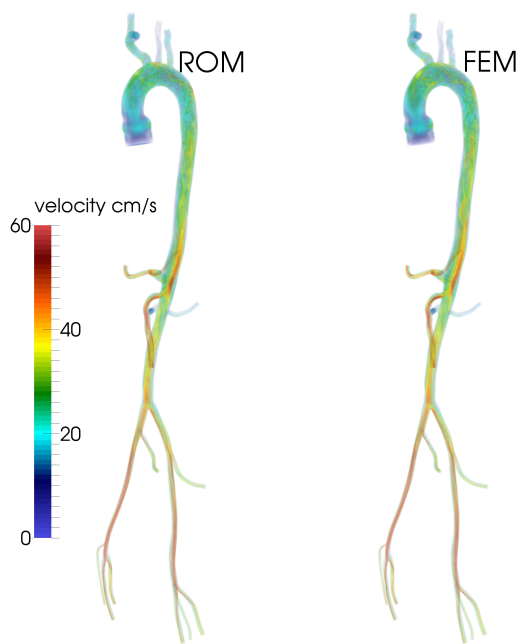

(d) time : $2.5 \mathrm{~s}$

Figure 14: Comparisons between velocity solutions computed for $E=4 \cdot 10^{6} \mathrm{dyn} / \mathrm{cm}^{2}$ using the reduced order model with 71 basis functions (left) and the finite element one (right). 


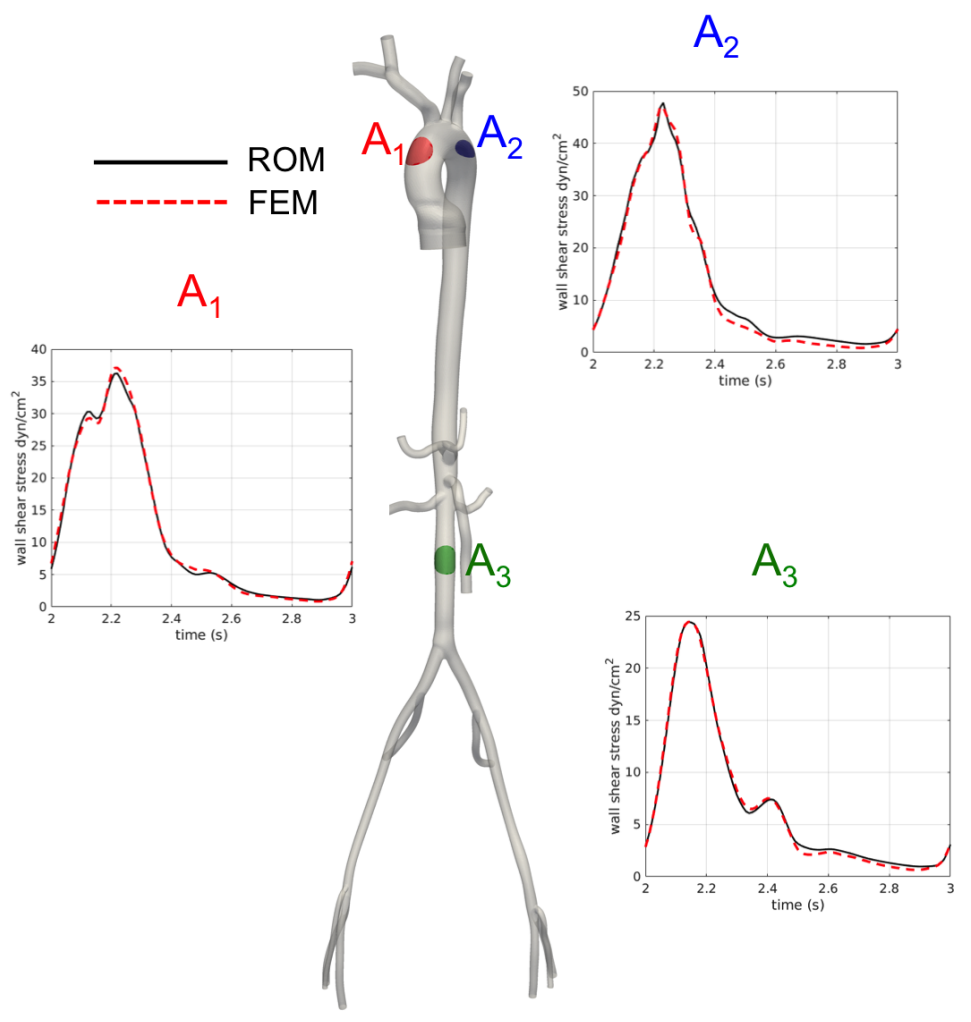

Figure 15: Comparisons between WSS computed for $E=4 \cdot 10^{6} \mathrm{dyn} / \mathrm{cm}^{2}$ using the reduced order model with 71 basis functions (left) and the finite element one (right). 


\subsection{Comparisons between FSI and RFSI}

In this section we report a comparison of the results obtained using the 3D implicit FSI solver described in Section 2 against those generated by the RFSI one reported in Section 3. In particular, we focus on the outlet mean pressures, velocity profiles at several locations and wall shear stress quantities.

In Figure 16 we compare the time evolution of the fluid pressure at all the outflow sections. We notice that both solvers lead to comparable results in terms of pressure wave speed either in proximal and distal branches. By comparing the peak values of the pressure obtained we notice that they are in excellent agreement for what concerns the proximal arteries. Conversely, moving towards distal vessels, it is possible to observe a gap in the peak pressure values. We remark that these differences may be due to the dumping of the viscous term used in the RFSI solver for the Robin condition (5.2) used to model the surrounding tissue. Furthermore such an effect is visible in the early diastolic phase wherein the pressure oscillations are dumped out in the RFSI solver wrt to the 3D FSI one.

In figures 17 and 18 we compare the velocity profiles at time $2.15 \mathrm{~s}$ and $2.4 \mathrm{~s}$, respectively, at three locations of our domain. At time $2.15 \mathrm{~s}$, which corresponds to the systolic peak of the third heartbeat, both solvers produce velocity profiles that are in good agreement. Of course, the results obtained with the full 3D FSI feature a much higher resolution thanks to the smaller mesh size used. At time 2.4 s, i.e. during the mid-deceleration phase, we notice that the velocity profiles are qualitatively similar although the FSI solver, wherein the SUPG-VMS stabilization is used, is able to capture better local features of the blood flow dynamic like small vortexes.

Figure 19 shows a comparison of the Wall Shear Stresses (WSS). Specifically, we focus on three different patches located at the fluid-structure interface where we evaluate the time evolution of the WSS magnitude averaged by the patch area and its normalization by the corresponding temporal mean. The WSS curves obtained by both solvers are in agreement for what concerns their phase, expecially in $A_{1}$ and $A_{2}$ areas. During the systolic phase the WSS values obtained by the two solvers feature a remarkable difference. Similar comparisons have already been carried out in [24] where the case of an aortic arch was considered. Nevertheless, in the same work a better agreement was reported for other geometries (femoropopliteal bypass). This difference may come from the use of transpiration conditions (3.2) in the RFSI solver in presence of larger displacements. Indeed, transpiration conditions introduce a modelling error proportional to the displacement. In the comparisons reported here, the mismatch may be associated also with the different dicretization choices related to the two solvers concerning the fluid problem: in fact, in the FSI solver we used an isotropic grid and P1-P1 stabilized finite elements, while, in the RFSI solver we chose an anisotropic grid featuring a boundary layer on the fluid-structure interface and P2P1 finite elements. 


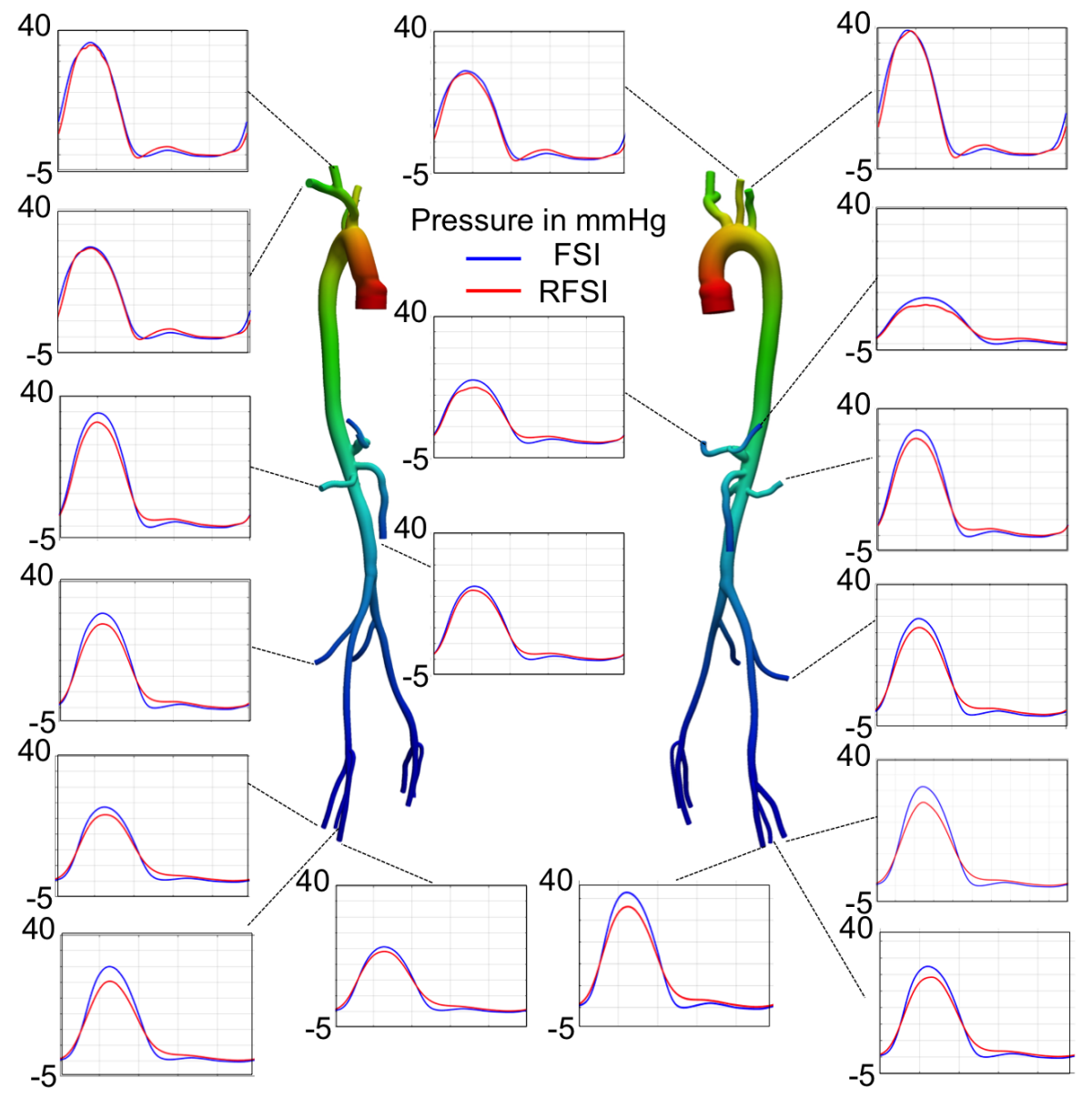

Figure 16: Comparisons between outflow mean pressures computed in the third hearbeat obtained by solving the 3D FSI and the RFSI problems. 


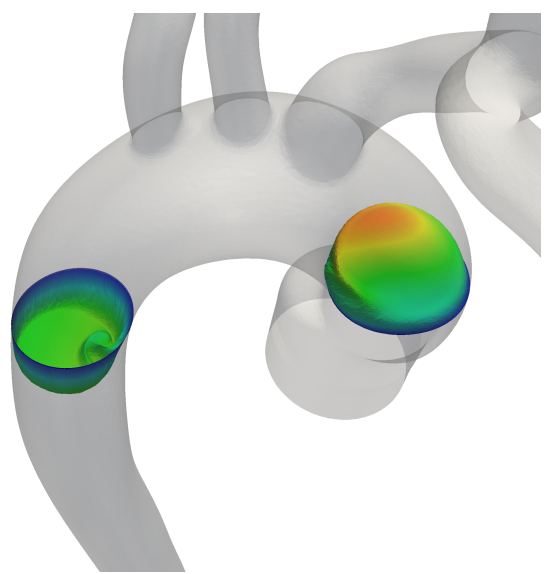

(a) FSI ,top

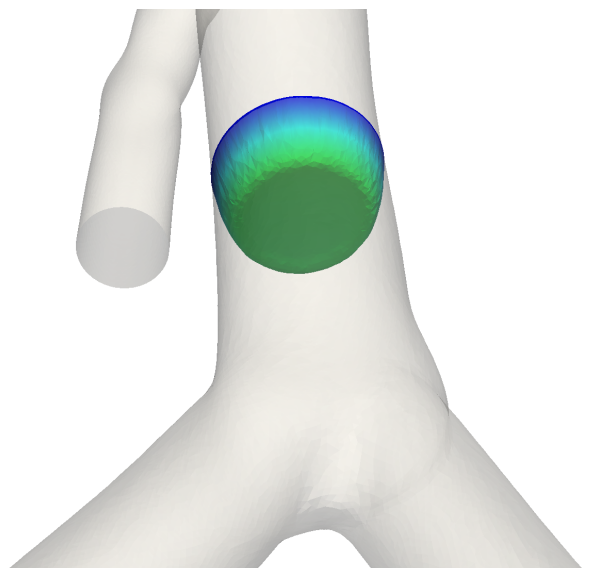

(c) FSI, bottom

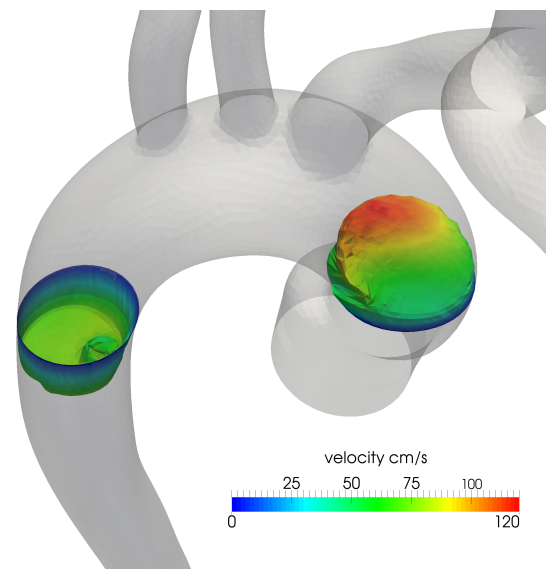

(b) RFSI, top

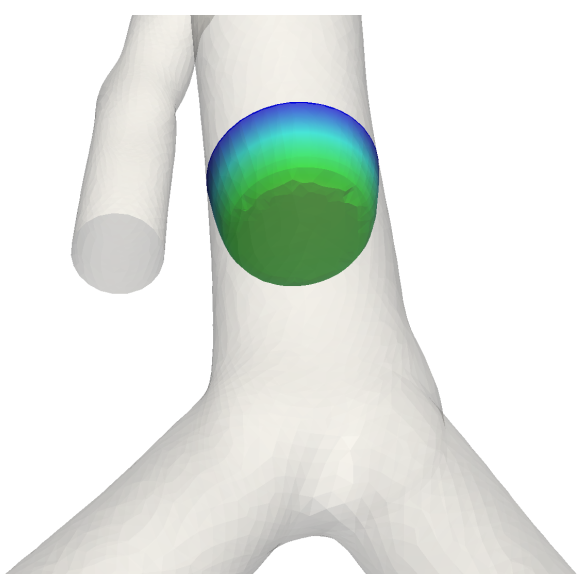

(d) RFSI, bottom

Figure 17: Comparisons between velocity profiles at time $2.15 \mathrm{~s}$. On the right, the solution is $\mathrm{P} 2$ but the visualisation is only P1. 


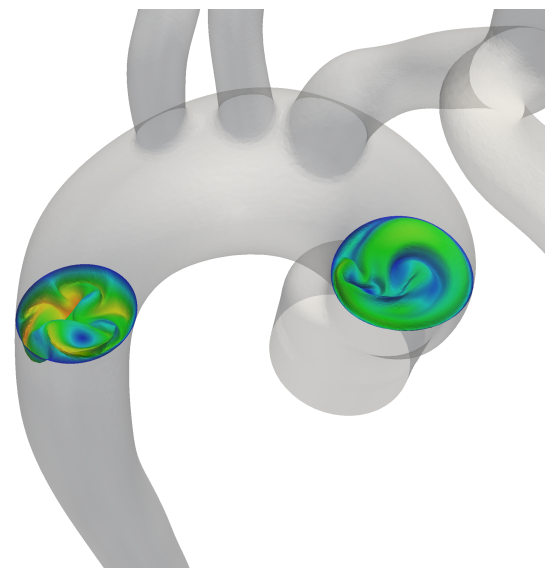

(a) FSI ,top

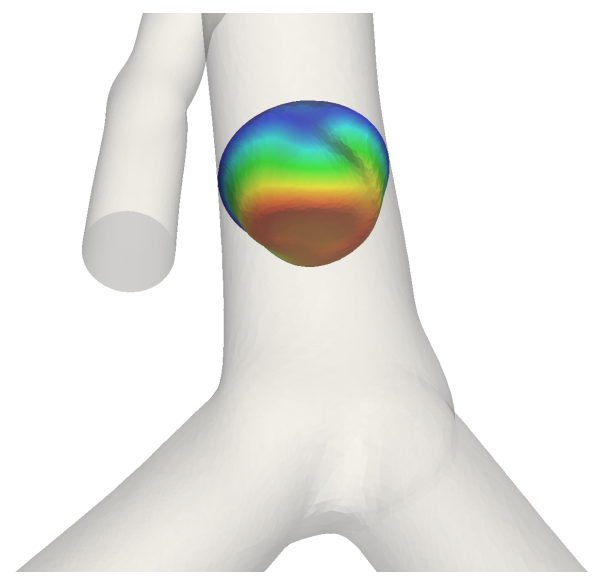

(c) FSI, bottom

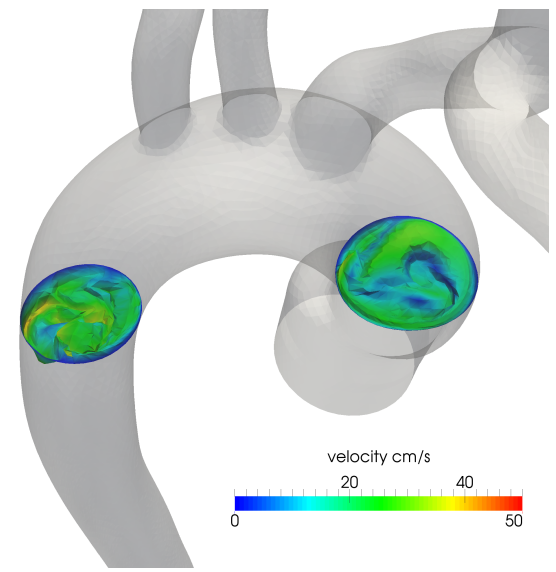

(b) RFSI, top

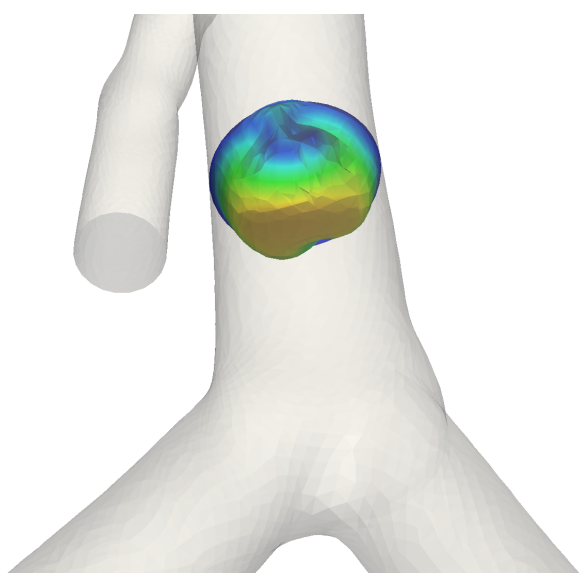

(d) RFSI, bottom

Figure 18: Comparisons between velocity profiles at time $2.4 \mathrm{~s}$. On the right, the solution is $\mathrm{P} 2$ but the visualisation is only P1. 

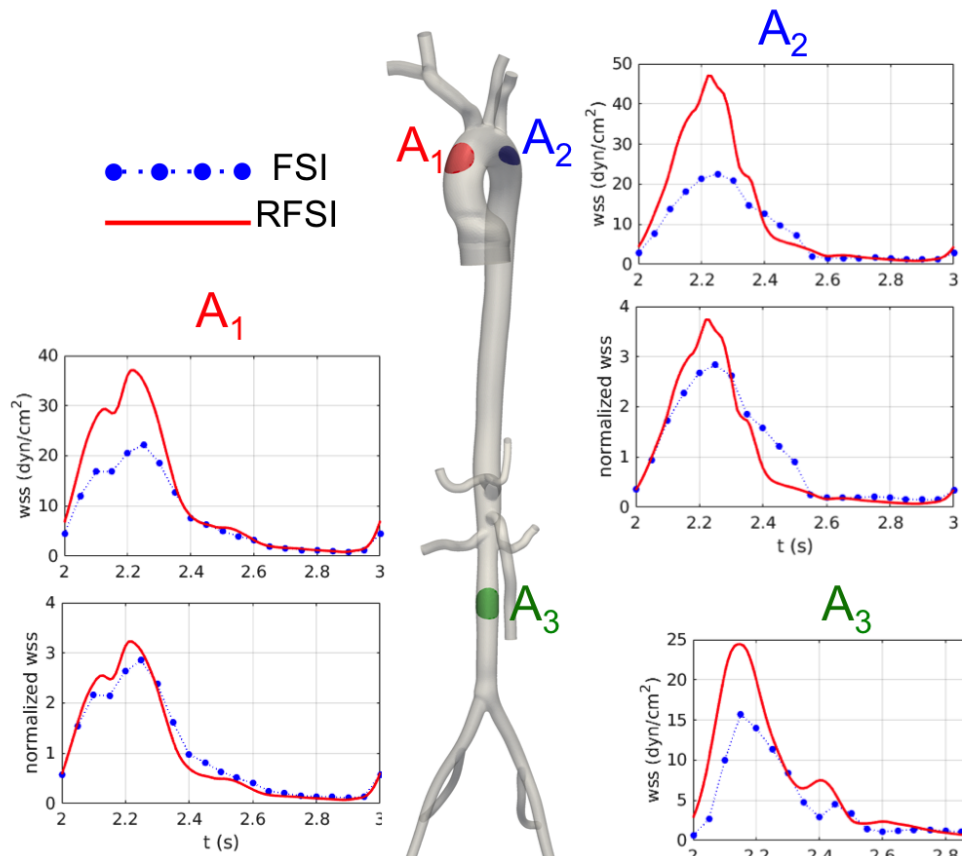

$\mathrm{A}_{3}$
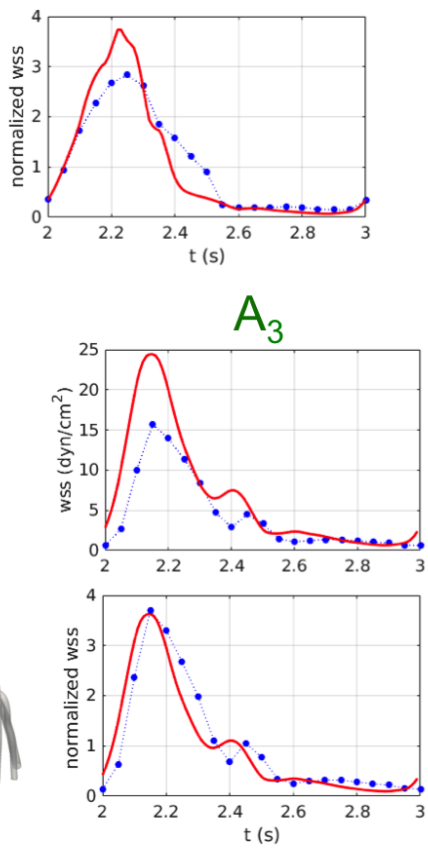

Figure 19: Comparisons between the magnitude of the wall shear stress averaged on three different areas obtained by solving the 3D FSI and the RFSI problems. 


\section{Conclusions}

When dealing with vascular flow simulation several modeling and numerical options are available. The choice depends on the targeted measures or indicators. In this work we have presented two possibilities, one relying on a detailed three dimensional FSI model for both the solid and the fluid, and one, RFSI, which focus on the fluid mechanics and allows for further numerical reduction. The FSI model is approximated by the finite element method and its resolution requires parallel methods, in particular we use a FaCSI preconditioner derived from domain decomposition and multigrid methods. RFSI relies on a fixed geometry and generalized Robin conditions to account for a membrane model. It is firstly approximated by the finite element method in an offline phase, then by POD and a Galerkin projection to a very small subspace in the online phase.

We have compared the two methods and showed that RFSI introduces modeling errors that has to be taken into account when interpreting the numerical results. However it allows for drastic reductions of the computational times and runs online almost in real time on a simple laptop. It is important to notice that the online solver is specialized for the geometry at hand: changing the geometry, for example a new patient, requires the recomputation of the offline phase.

Acknowledgments. The authors whish to thank Prof. Alfio Quarteroni for his menthoring on all the subjects of this paper, Andrea Bartezzaghi for the realization of a very efficient online solver which is used during the presentations, and the whole LifeV community.

\section{Bibliography}

[1] Open Source Medical Software Corporation, http://www.vascularmodel.com/ sandbox/doku.php.

[2] P. R. Amestoy, I. S. Duff, J. Koster and J. Y. L'Excellent, A fully asynchronous multifrontal solver using distributed dynamic scheduling, SIAM Journal on Matrix Analysis and Applications 23 (2001), 15-41.

[3] P. R. Amestoy, A. Guermouche, J. Y. L'Excellent and S. Pralet, Hybrid scheduling for the parallel solution of linear systems, Parallel Computing 32 (2006), 136-156.

[4] S. Badia, F. Nobile and C. Vergara, Fluid-structure partitioned procedures based on Robin transmission conditions, Journal of Computational Physics 227 (2008), 70277051 .

[5] _ Robin-Robin preconditioned Krylov methods for fluid-structure interaction problems, Computer Methods in Applied Mechanics and Engineering 198 (2009), 2768-2784.

[6] S. Badia, A. Quaini and A. Quarteroni, Modular vs. non-modular preconditioners for fluid-structure systems with large added-mass effect, Computer Methods in Applied Mechanics and Engineering 197 (2008), 4216-4232. 
[7] S. Badia, A. Quaini and A. Quarteroni, Modular vs. non-modular preconditioners for fluid-structure systems with large added-mass effect, Computer Methods in Applied Mechanics and Engineering 197 (2008), 4216-4232.

[8] S. Badia, A. Quaini and A. Quarteroni, Splitting methods based on algebraic factorization for fluid-structure interaction, SIAM Journal on Scientific Computing 30 (2008), $1778-1805$.

[9] F. Ballarin, E. Faggiano, S. Ippolito, A. Manzoni, A. Quarteroni, G. Rozza and R. Scrofani, Fast simulations of patient-specific haemodynamics of coronary artery bypass grafts based on a POD-Galerkin method and a vascular shape parametrization, Journal of Computational Physics 315 (2016), 609 - 628.

[10] F. Ballarin and G. Rozza, POD-Galerkin monolithic reduced order models for parametrized fluid-structure interaction problems, International Journal for Numerical Methods in Fluids (2016), n/a-n/a, fld.4252.

[11] A. T. Barker, Parallel monolithic fluid-structure interaction algorithms with application to blood flow simulation, Ph.D. thesis, University of Colorado, 2009.

[12] Andrew T. Barker and Xiao-Chuan Cai, Scalable parallel methods for monolithic coupling in fluid-structure interaction with application to blood flow modeling, Journal of Computational Physics 229 (2010), 642-659.

[13] Y. Bazilevs, V. M. Calo, J. A. Cottrell, T. J. R. Hughes, A. Reali and G. Scovazzi, Variational multiscale residual-based turbulence modeling for large eddy simulation of incompressible flows, Computer Methods in Applied Mechanics and Engineering 197 (2007), 173-201.

[14] Y. Bazilevs, V. M. Calo, T. J. R. Hughes and Y. Zhang, Isogeometric fluid-structure interaction: theory, algorithms, and computations, Computational Mechanics 43 (2008), $3-37$.

[15] Y. Bazilevs, K. Takizawa and T. E. Tezduyar, Computational fluid-structure interaction: methods and applications, John Wiley \& Sons, 2013.

[16] L. Bertagna, L. Formaggia, S. Deparis, D. Forti and A. Veneziani, The LifeV library: engineering mathematics beyond the proof of concept, submitted, 2016.

[17] L. Bertagna and A. Veneziani, A model reduction approach for the variational estimation of vascular compliance by solving an inverse fluid-structure interaction problem, Inverse Problems 30 (2014), 055006.

[18] D. Boffi, N. Cavallini and L. Gastaldi, Finite element approach to immersed boundary method with different fluid and solid densities, Mathematical Models and Methods in Applied Sciences 21 (2011), 2523-2550.

[19] L. Boussel, V. Rayz, C. McCulloch, A. Martin, G. Acevedo-Bolton, M. Lawton, R. Higashida, W. S. Smith, W. L. Young and D. Saloner, Aneurysm Growth Occurs at Region of Low Wall Shear Stress, Stroke 39 (2008), 2997-3002.

[20] J. Burkardt, M. Gunzburger and H.-C. Lee, POD and CVT-based reduced-order modeling of Navier-Stokes flows, Computer Methods in Applied Mechanics and Engineering 196 (2006), $337-355$. 
[21] A. Caiazzo, R. Guibert and I. E. Vignon-Clementel, A reduced-order modeling for efficient design study of artificial valve in enlarged ventricular outflow tracts, Computer Methods in Biomechanics and Biomedical Engineering 19 (2016), 1314-1318.

[22] P. Causin, J. F. Gerbeau and F. Nobile, Added-mass effect in the design of partitioned algorithms for fluid-structure problems, Computer Methods in Applied Mechanics and Engineering 194 (2005), 4506-4527.

[23] C. M. Colciago, Reduced Order Fluid-Structure Interaction Models for Haemodynamics Applications, Ph.D. thesis, SB, Lausanne, 2014.

[24] C. M. Colciago, S. Deparis and A. Quarteroni, Comparisons between reduced order models and full 3D models for fluid-structure interaction problems in haemodynamics, J. Comput. Appl. Math. 265 (2014), 120 - 138, Current Trends and Progresses in Scientific Computation Dedicated to Professor Ben-yu Guo on His 70th Birthday.

[25] G.-H. Cottet, E. Maitre and T. Milcent, Eulerian formulation and level set models for incompressible fluid-structure interaction, Mathematical Modelling and Numerical Analysis 42 (2008), 471-492.

[26] P. Crosetto, S. Deparis, G. Fourestey and A. Quarteroni, Parallel Algorithms for FluidStructure Interaction Problems in Haemodynamics, SIAM Journal on Scientific Computing 33 (2011), 1598-1622.

[27] P. Crosetto, S. Deparis, G. Fourestey and A. Quarteroni, Parallel Algorithms for FluidStructure Interaction Problems in Haemodynamics, SIAM Journal on Scientific Computing 33 (2011), 1598-1622.

[28] J. Degroote, K.-J. Bathe and J. Vierendeels, Performance of a new partitioned procedure versus a monolithic procedure in fluid-structure interaction, Computers \& Structures 87 (2009), 793-801.

[29] S. Deparis, M. Discacciati, G. Fourestey and Alfio Quarteroni, Fluid-structure algorithms based on Steklov-Poincaré operators, Computer Methods in Applied Mechanics and Engineering 195 (2006), 5797-5812.

[30] S. Deparis, M. Discacciati and A. Quarteroni, A Domain Decomposition Framework for Fluid-Structure Interaction Problems, Computational Fluid Dynamics 2004 (Clinton Groth and DavidW. Zingg, eds.), Springer Berlin Heidelberg, 2006, pp. 41-58 (English).

[31] S. Deparis, M. A. Fernandez and L. Formaggia, Acceleration of a fixed point algorithm for fluid-structure interaction using transpiration conditions, M2AN Mathematical Modelling and Numerical Analysis. 37 (2003), 601-616.

[32] S. Deparis, D. Forti, G. Grandperrin and A. Quarteroni, FaCSI: A Block Parallel Preconditioner for Fluid-Structure Interaction in Hemodynamics, Journal of Computational Physics (accepted), 2016.

[33] S. Deparis, D. Forti, A. Heinlein, A. Klawonn, A. Quarteroni and O. Rheinbach, A comparison of Preconditioners for the Steklov-Poincaré Formulation of the Fluid-Structure Coupling in Hemodynamics, Proceedings in Applied Mathematics and Mechanics 15 (2015), 93-94. 
[34] S. Deparis, G. Grandperrin and A. Quarteroni, Parallel preconditioners for the unsteady Navier-Stokes equations and applications to hemodynamics simulations, Computers \& Fluids 92 (2014), 253 - 273.

[35] W. G. Dettmer and D. Perić, A Fully Implicit Computational Strategy for Strongly Coupled Fluid-Solid Interaction, Archives of Computational Methods in Engineering 14 (2007), 205-247 (English).

[36] E. S. Di Martino and D. A. Vorp, Effect of Variation in Intraluminal Thrombus Constitutive Properties on Abdominal Aortic Aneurysm Wall Stress, Annals of Biomedical Engineering 31 (2003), 804-809.

[37] J. Donea, S. Giuliani and J.P. Halleux, An Arbitrary Lagrangian-Eulerian Finite Element Method for Transient Dynamic Fluid-Structure Interaction, Computer Methods in Applied Mechanics and Engineering 33 (1982), 689-723.

[38] T. Dunne, Adaptive Finite Element Approximation of Fluid-Structure Interaction Based on Eulerian and Arbitrary Lagrangian-Eulerian Variational Formulations, Ph.D. thesis, Ruprechts-Karls Universität Heidelberg, 2007.

[39] E. Faggiano and L. Antiga, An open-source tool for patient-specific fluid-structure vessel mesh generation, (2015), In preparation.

[40] M. A. Fernandez, J.F. Gerbeau and C. Grandmont, A projection semi-implicit scheme for the coupling of an elastic structure with an incompressible fluid, International Journal for Numerical Methods in Engineering 64 (2007), 794-821.

[41] M. A. Fernández and M. Moubachir, An exact block-Newton algorithm for solving fluid-structure interaction problems, C. R. Math. Acad. Sci. Paris 336 (2003), 681-686.

[42] M. A. Fernández, J. Mullaert and M. Vidrascu, Generalized Robin-Neumann explicit coupling schemes for incompressible fluid-structure interaction: Stability analysis and numerics, International Journal for Numerical Methods in Engineering 101 (2015), 199-229.

[43] C. A. Figueroa, I. E. Vignon-Clementel, K. E. Jansen, T. J.R. Hughes and C. A. Taylor, A coupled momentum method for modeling blood flow in three-dimensional deformable arteries, Computer Methods in Applied Mechanics and Engineering 195 (2006), 5685-5706.

[44] L. Formaggia, A. Quarteroni and A. Veneziani, Cardiovascular mathematics, Springer, 2009.

[45] L. Formaggia, A. Quarteroni and A. Veneziani (eds.), Cardiovascular mathematics, MS\&A. Modeling, Simulation and Applications 1, Springer-Verlag Italia, Milan, 2009, Modeling and simulation of the circulatory system.

[46] D. Forti and L. Dedè, Semi-implicit BDF time discretization of the Navier-Stokes equations with VMS-LES modeling in High Performance Computing framework, Computers \& Fluids 117 (2015), 168-182.

[47] D. Forti, A. Quarteroni and S. Deparis, A parallel algorithm for the solution of largescale non-conforming fluid-structure interaction problems in hemodynamics, Submitted, 2015. 
[48] T. C. Gasser, R. W. Ogden and G. A Holzapfel, Hyperelastic modelling of arterial layers with distributed collagen fibre orientations, Journal of The Royal Society Interface 3 (2006), 15-35.

[49] M. W. Gee, U. Küttler and W. A. Wall, Truly monolithic algebraic multigrid for fluidstructure interaction, International Journal for Numerical Methods in Engineering 26 (2010), 52-72.

[50] J. F. Gerbeau and M. Vidrascu, A quasi-Newton algorithm based on a reduced model for fluid-structure interaction problems in blood flows, Mathematical Modelling and Numerical Analysis 37 (2003), 631-647.

[51] A.-L. Gerner, A. Reusken and K. Veroy, Reduced Basis A Posteriori Error Bounds for the Instationary Stokes Equations, submitted, 2012.

[52] A.-L. Gerner and K. Veroy, Reduced basis a posteriori error bounds for the Stokes equations in parametrized domains: a penalty approach, Mathematical Models and Methods in Applied Sciences 21 (2011), 2103-2134.

[53] A. Gerstenberger and W. A. Wall, An extended finite element method/Lagrange multiplier based approach for fluid-structure interaction, Computer Methods in Applied Mechanics and Engineering 197 (2008), 1699-1714.

[54] P. Gervasio, F. Saleri and A. Veneziani, Algebraic fractional-step schemes with spectral methods for the incompressible Navier-Stokes equations, Journal of Computational Physics 214 (2006), 347-365.

[55] L. Grinberg and G. E. Karniadakis, Outflow boundary conditions for arterial networks with multiple outlets, Annals of biomedical engineering 36 (2008), 1496-1514.

[56] L. Grinberg, A. Yakhot and G. Karniadakis, Analyzing Transient Turbulence in a Stenosed Carotid Artery by Proper Orthogonal Decomposition, Annals of Biomedical Engineering 37 (2009), 2200-2217 (English).

[57] R. Guibert, K. McLeod, A. Caiazzo, T. Mansi, M. A. Fernéndez, M. Sermesant, X. Pennec, I. E. Vignon-Clementel, Y. Boudjemline and J.-F. Gerbeau, Group-wise construction of reduced models for understanding and characterization of pulmonary blood flows from medical images, Medical Image Analysis 18 (2014), 63 - 82.

[58] M. D. Gunzburger, J. S. Peterson and J. N. Shadid, Reduced-order modeling of timedependent PDEs with multiple parameters in the boundary data, Computer Methods in Applied Mechanics and Engineering 196 (2007), 1030 - 1047.

[59] M. Heil, An efficient solver for the fully coupled solution of large-displacement fluidstructure interaction problems, Computer Methods in Applied Mechanics and Engineering 193 (2004), 1-23.

[60] M. Heil, A. L. Hazel and J. Boyle, Solvers for large-displacement fluid-structure interaction problems: segregated versus monolithic approaches, Computational Mechanics 43 (2008), 91-101 (English).

[61] J. S Hesthaven, G. Rozza and B. Stamm, Certified Reduced Basis Methods for Parametrized Partial Differential Equations, 1 ed, Springer International Publishing, The address, 2016. 
[62] G. A. Holzapfel, T. C. Gasser and R. W. Ogden, A New Constitutive Framework for Arterial Wall Mechanics and a Comparative Study of Material Models, Journal of elasticity and the physical science of solids 61 (2000), 1-48.

[63] G. A Holzapfel and R. W Ogden, Mechanics of biological tissue, Springer Science \& Business Media, 2006.

[64] J. Hron and S. Turek, A monolithic FEM/Multigrid solver for an ALE formulation of fluid-structure interaction with applications in biomechanics, in: Fluid-structure interaction (H.-J. Bungartz and M. Schäfer, eds.), Lecture Notes in Computational Science and Engineering 53, pp. 146-170, 2006.

[65] T. J.R. Hughes, W.K. Liu and T.K. Zimmermann, Lagrangian-Eulerian finite element formulation for incompressible viscous flow, Computer Methods in Applied Mechanics and Engineering 29 (1981), 329-349.

[66] J. D. Humphrey and K. R. Rajagopal, A constrained mixture model for growth and remodeling of soft tissues, Mathematical Models and Methods in Applied Sciences 12 (2002), 407-430.

[67] L. Iapichino, A. Quarteroni and G. Rozza, A reduced basis hybrid method for the coupling of parametrized domains represented by fluidic networks, Computer Methods in Applied Mechanics and Engineering 221-222 (2012), 63 - 82.

[68] Bärbel Janssen and Thomas Wick, Block preconditioning with SCHUR complements for monolithic fluid-structure interactions, in: Proceedings of $V$ European Conference on Computational Fluid Dynamics ECCOMAS CFD 2010, Lisbon, Portugal,, June 14172010.

[69] L.-D. Jou, D. H. Lee, H. Morsi and M. E. Mawad, Wall Shear Stress on Ruptured and Unruptured Intracranial Aneurysms at the Internal Carotid Artery, AJNR Am J Neuroradiol 29 (2008), 1761-1767.

[70] T. Kashiwabara, C. Colciago, L. Dedé and A. Quarteroni, Well-posedness, regularity, and convergence analysis of the finite element approximation of a generalized Robin boundary value problem, submitted, 2014.

[71] E. Kuhl, R. Maas, G. Himpel and A. Menzel, Computational modeling of arterial wall growth, Biomechanics and Modeling in Mechanobiology 6 (2007), 321-331.

[72] K. Kunisch and S. Volkwein, Galerkin Proper Orthogonal Decomposition Methods for a General Equation in Fluid Dynamics, SIAM Journal on Numerical Analysis 40 (2003), pp. 492-515 (English).

[73] U. Küttler, M. W. Gee, C. Förster, A. Comerford and W. A. Wall, Coupling strategies for biomedical fluid-structure interaction problems, International Journal for Numerical Methods in Biomedical Engineering 26 (2010), 305-321.

[74] U. Küttler and W. A. Wall, The Dilemma of Domain Decomposition Approaches in Fluid-Structure Interactions with Fully Enclosed Incompressible Fluids, Domain Decomposition Methods in Science and Engineering XVII (Ulrich Langer, Marco Discacciati, DavidE. Keyes, OlofB. Widlund and Walter Zulehner, eds.), Lecture Notes in Computational Science and Engineering 60, Springer Berlin Heidelberg, 2008, pp. 575582 (English). 
[75] putational Mechanics 43 (2008), 61-72.

[76] U. Langer and H. Yang, Domain Decomposition Solvers for Some Fluid-Structure Interaction Problems, Proceedings in Applied Mathematics and Mechanics 12 (2012), $375-376$.

[77] _ Partitioned solution algorithms for fluid-structure interaction problems with hyperelastic models, Journal of Computational and Applied Mathematics 276 (2015), $47-61$.

[78] T. Lassila, A. Manzoni, A. Quarteroni and G. Rozza, Boundary control and shape optimization for the robust design of bypass anastomoses under uncertainty, ESAIM: Mathematical Modelling and Numerical Analysis 47 (2013), 1107-1131.

[79] P. Le Tallec and J. Mouro, Fluid structure interaction with large structural displacements, Computer Methods in Applied Mechanics and Engineering 190 (2001), 30393067.

[80] F. Loth, P. F. Fischer and H. S. Bassiouny, Blood flow in end-to-side anastomoses, Annual Review of Fluid Mechanics 40 (2008), 367-393.

[81] F. Loth, S. A. Jones, C. K. Zarins, D. P. Giddens, R. F. Nassar, S. Glagov and H. S. Bassiouny, Relative contribution of wall shear stress and injury in experimental intimal thickening at PTFE end-to-side arterial anastomoses, J Biomech Eng 124 (2002), 44-51.

[82] Malek A. M., Alper S. L. and Izumo S, Hemodynamic shear stress and its role in atherosclerosis, JAMA 282 (1999), 2035-2042.

[83] A.C.I. Malossi and J. Bonnemain, Numerical Comparison and Calibration of Geometrical Multiscale Models for the Simulation of Arterial Flows, Cardiovascular Engineering and Technology 4 (2013), 440-463 (English).

[84] A. Manzoni, A. Quarteroni and G. Rozza, Model reduction techniques for fast blood flow simulation in parametrized geometries, International Journal for Numerical Methods in Biomedical Engineering 28 (2012), 604-625.

[85] A. Manzoni, A. Quarteroni and G. Rozza, Shape optimization for viscous flows by reduced basis methods and free-form deformation, International Journal for Numerical Methods in Fluids 70 (2012), 646-670.

[86] H. G. Matthies, R. Niekamp and J. Steindorf, Algorithms for strong coupling procedures, Computer Methods in Applied Mechanics and Engineering 195 (2006), 20282049.

[87] U. M. Mayer, A. Popp, A. Gerstenberger and W. A. Wall, 3D fluid-structure-contact interaction based on a combined XFEM FSI and dual mortar contact approach, Computational Mechanics 46 (2010), 53-67 (English).

[88] C. Michler, S. J. Hulshoff, E. H. Van Brummelen and R. De Borst, A monolithic approach to fluid-structure interaction, Computers \& fluids 33 (2004), 839-848.

[89] R. Mittal and G. Iaccarino, Immersed boundary methods, Annual review of fluid mechanics. Vol. 37, Annu. Rev. Fluid Mech. 37, Annual Reviews, Palo Alto, CA, 2005, pp. 239-261. 
[90] P. Moireau, N. Xiao, M. Astorino, C. A. Figueroa, D.Chapelle, C. A. Taylor and J.F. Gerbeau, External tissue support and fluid-structure simulation in blood flows, Biomechanics and Modeling in Mechanobiology 11 (2012), 1-18.

[91] N. M. Newmark, A method of computation for structural dynamics, Journal of the Engineering Mechanics Division 85 (1959), 67-94.

[92] W. W Nichols and M. F. O'Rourke, McDonald's Blood Flow in Arteries - Theoretical, experimental and clinical principles -, Arnold, 1998.

[93] F. Nobile., Numerical approximation of fluid-structure interaction problems with application to haemodynamics, Ph.D. thesis, EPFL, 2001.

[94] F. Nobile, Coupling strategies for the numerical simulation of blood flow in deformable arteries by 3D and 1D models, Mathematical and Computer Modelling 49 (2009), 2152 - 2160, Trends in Application of Mathematics to Medicine Trends in Application of Mathematics to Medicine.

[95] F. Nobile, M. Pozzoli and C. Vergara, Inexact accurate partitioned algorithms for fluidstructure interaction problems with finite elasticity in haemodynamics, Journal of Computational Physics 273 (2014), 598 - 617.

[96] R. W. Ogden, Anisotropy and Nonlinear Elasticity in Arterial Wall Mechanics, pp. 179258, Springer Vienna, Vienna, 2009.

[97] T. G. Papaioannou, E. N. Karatzis, M. Vavuranakis, J. P. Lekakis and C. Stefanadis, Assessment of vascular wall shear stress and implications for atherosclerotic disease, International Journal of Cardiology 113 (2006), 12 - 18.

[98] C. S. Peskin, The immersed boundary method, Acta Numerica 11 (2002), 479-517.

[99] A. Quarteroni, A. Manzoni and F. Negri, Reduced Basis Methods for Partial Differential Equations, 1 ed, 92, Springer International Publishing, 2016.

[100] A. Quarteroni and G. Rozza (eds.), Reduced Order Methods for Modeling and Computational Reduction, MS\&A, Modeling, Simulation and Applications, Springer, Milano, 2013, Book edited with selected contributions from CECAM workshop, EPFL, May 2012.

[101] R. Rannacher and T. Richter, An Adaptive Finite Element Method for Fluid-Structure Interaction Problems Based on a Fully Eulerian Formulation, Fluid Structure Interaction II (H.-J. Bungartz et al., ed.), Springer-Verlag Berlin Heidelberg, 2010, Lecture Notes in Computational Science and Engineering 73, pp. 159-192.

[102] T. Richter, A fully Eulerian formulation for fluid-structure-interaction problems with large deformations and free structure movement, in: Proceedings of the V European Conference on Computational Fluid Dynamics ECCOMAS CFD 2010, Lisbon, Portugal, 14-17 June 2010, 2010.

[103] T. Richter, A Fully Eulerian formulation for fluid-structure-interaction problems, Journal of Computational Physics 233 (2013), 227-240.

[104] C. W. Rowley, Model reduction for fluids, using balanced Proper Orthogonal Decomposition, International Journal of Bifurcation and Chaos (IJBC) 15 (2005), 997-1013. 
[105] G. Rozza, D.B.P. Huynh and A. Manzoni, Reduced basis approximation and a posteriori error estimation for Stokes flows in parametrized geometries: roles of the inf-sup stability constants, Numer. Math. 125 (2013), 115-152.

[106] G. Rozza, A. Manzoni and F. Negri, Reduction strategies for PDE-constrained optimization probems in Haemodynamics, in: European Congress on Computational Methods in Applied Sciences and Engineering ( $\mathrm{J}$ et al. Eberhardsteiner, ed.), pp. 1-20, September 10-14 2012.

[107] G. Rozza and K. Veroy, On the stability of the reduced basis method for Stokes equations in parametrized domains, Computer Methods in Applied Mechanics and Engineering 196 (2007), 1244 - 1260.

[108] M. Shojima, M. Oshima, K. Takagi, R. Torii, M. Hayakawa, K. Katada, A. Morita and T. Kirino, Magnitude and role of wall shear stress on cerebral aneurysm: computational fluid dynamic study of 20 middle cerebral artery aneurysms, Stroke 35 (2004), 2500-5.

[109] M. Souli, A. Ouahsine and L. Lewin, \{ALE\} formulation for fluid-structure interaction problems, Computer Methods in Applied Mechanics and Engineering 190 (2000), 659 -675 .

[110] K. Stein, T. Tezduyar and R. Benney, Mesh moving techniques for fluid-structure interactions with large displacements, Journal of Applied Mechanics 70 (2003), 58-63.

[111] K. Takizawa and T. E. Tezduyar, Multiscale space-time fluid-structure interaction techniques, Computational Mechanics 48 (2011), 247-267.

[112] C.A. Taylor and J.D. Humphrey, Open problems in computational vascular biomechanics: Hemodynamics and arterial wall mechanics, Computer Methods in Applied Mechanics and Engineering 198 (2009), 3514 - 3523, Models and Methods in Computational Vascular and Cardiovascular Mechanics.

[113] T. E. Tezduyar and S. Sathe, Modelling of fluid-structure interactions with the spacetime finite elements: solution techniques, International Journal for Numerical Methods in Fluids 54 (2007), 855-900.

[114] T. E. Tezduyar, S. Sathe, M. Schwaab and B. S. Conklin, Arterial fluid mechanics modeling with the stabilized space-time fluid-structure interaction technique, International Journal for Numerical Methods in Fluids 57 (2008), 601-629.

[115] T. E. Tezduyar, S. Sathe and K. Stein, Solution techniques for the fully discretized equations in computation of fluid-structure interactions with the space-time formulations, Computer Methods in Applied Mechanics and Engineering 195 (2006), 5743-5753.

[116] J. Tong, T. Cohnert, P. Regitnig and G.A. Holzapfel, Effects of Age on the Elastic Properties of the Intraluminal Thrombus and the Thrombus-covered Wall in Abdominal Aortic Aneurysms: Biaxial Extension Behaviour and Material Modelling, European Journal of Vascular and Endovascular Surgery 42 (2011), 207 - 219.

[117] A. Toselli and O. Widlund, Domain decomposition methods: algorithms and theory, Springer, 2005.

[118] P. Tricerri, L. Dedè, S. Deparis, A. Quarteroni, A. M. Robertson and A. Sequeira, Fluid-structure interaction simulations of cerebral arteries modeled by isotropic and anisotropic constitutive laws, Computational Mechanics 55 (2015), 479-498. 
[119] A. Valencia and F. Solis, Blood flow dynamics and arterial wall interaction in a saccular aneurysm model of the basilar artery, Computers \& Structures 84 (2006), 1326 - 1337.

[120] E. H. Van Brummelen, Added mass effects of compressible and incompressible flows in fluid-structure interaction, Journal of Applied mechanics 76 (2009), 021206-1-0212067.

[121] _ Partitioned iterative solution methods for fluid-structure interaction, International Journal for Numerical Methods in Fluids 65 (2011), 3-27.

[122] W. A. Wall, A. Gerstenberger, P. Gamnitzer, C. Förster and E. Ramm, Large deformation fluid-structure interaction-advances in ALE methods and new fixed grid approaches, Fluid-structure interaction, Springer, 2006, pp. 195-232.

[123] W. A. Wall, A. Gerstenberger, U. Küttler and U. M. Mayer, An XFEM Based Fixed-Grid Approach for 3D Fluid-Structure Interaction, Fluid Structure Interaction II (H.-J. Bungartz et al., ed.), Springer-Verlag Berlin Heidelberg, 2010, Lecture Notes in Computational Science and Engineering 73, pp. 327-349.

[124] H. Wang, J. Chessa, W. K. Liu and T. Belytschko, The immersed/fictitious element method for fluid-structure interaction: volumetric consistency, compressibility and thin members, International Journal for Numerical Methods in Engineering 74 (2008), 32 55 .

[125] T. Wiesner, Flexible Aggregation-based Algebraic Multigrid Methods for Contact and Flow Problems, Phd thesis, Technische Universität München, 2015.

[126] K. Willcox and J. Peraire, Balanced model reduction via the proper orthogonal decomposition, AIAA Journal (2002), 2323-2330.

[127] Y. Wu and X.-C. Cai, A fully implicit domain decomposition based ALE framework for three-dimensional fluid-structure interaction with application in blood flow computation, Journal of Computational Physics 258 (2014), 524-537.

\section{Author information}

Claudia M. Colciago, MATHICSE, Chair of Modelling and Scientific Computing, École

Polytechnique Fédérale de Lausanne, Station 8, CH-1015 Lausanne, Switzerland.

E-mail: claudia.colciago@epfl.ch

Simone Deparis, MATHICSE, Chair of Modelling and Scientific Computing, École Polytechnique Fédérale de Lausanne, Station 8, CH-1015 Lausanne, Switzerland. E-mail: simone.deparis@epfl.ch

Davide Forti, MATHICSE, Chair of Modelling and Scientific Computing, École Polytechnique Fédérale de Lausanne, Station 8, CH-1015 Lausanne, Switzerland. E-mail: davide.forti@epfl.ch 